\title{
GENERALISING THE GHS ATTACK ON THE ELLIPTIC CURVE DISCRETE LOGARITHM PROBLEM
}

\section{F. HESS}

\section{Abstract}

The Weil descent construction of the GHS attack on the elliptic curve discrete logarithm problem (ECDLP) is generalised in this paper, to arbitrary Artin-Schreier extensions. A formula is given for the characteristic polynomial of Frobenius for the curves thus obtained, as well as a proof that the large cyclic factor of the input elliptic curve is not contained in the kernel of the composition of the conorm and norm maps. As an application, the number of elliptic curves that succumb to the basic GHS attack is considerably increased, thereby further weakening curves over $\mathbb{F}_{2155}$. Other possible extensions or variations of the GHS attack are discussed, leading to the conclusion that they are unlikely to yield further improvements.

\section{Introduction}

The Weil descent technique, proposed by Frey [7], provides a way of mapping the discrete logarithm problem on an elliptic curve (ECDLP) over a large finite field $\mathbb{F}_{q^{n}}$ to a discrete logarithm problem on a higher-dimensional abelian variety defined over the small finite field $\mathbb{F}_{q}$. Using this technique, it became feasible to study possible further constructions of such abelian varieties, as well as the hardness of the discrete logarithm problem on such varieties.

This was subsequently done by Galbraith and Smart [10], and by Gaudry, Hess and Smart [13], in even characteristic (that is, for $q$ a power of 2). The construction of [13] yields a very efficient algorithm to reduce the ECDLP to the discrete logarithm in the divisor class group of a hyperelliptic curve over $\mathbb{F}_{q}$. Since subexponential algorithms exist for the discrete logarithm problem in high-genus hyperelliptic curves, this gives a possible method of attack against the ECDLP. We refer to the method of [13] as the GHS attack.

Menezes and Qu [20] analyzed the GHS attack in some detail, and demonstrated that it does not apply to the case when $q=2$ and $n$ is prime. Since this is the common case in real-world applications, the work of Menezes and Qu means that the GHS attack does not apply to most of the systems that have actually been deployed. However, there are a few deployed elliptic curve systems that use the fields $\mathbb{F}_{2^{155}}$ and $\mathbb{F}_{2^{185}}$; see [17]. Hence there is considerable interest as to whether the GHS attack makes all curves over these fields vulnerable. In [24], Smart examined the GHS attack for elliptic curves with respect to the field extension $\mathbb{F}_{2}{ }^{155} / \mathbb{F}_{231}$, and concluded that such a technique is unlikely to work for any curve defined over $\mathbb{F}_{2155}$.

Jacobson, Menezes and Stein [18] also examined the field $\mathbb{F}_{2155}$, this time using the GHS attack down to the subfield $\mathbb{F}_{2^{5}}$. They concluded that such a strategy could be used in 
practice to attack around $2^{33}$ isomorphism classes of elliptic curves defined over $\mathbb{F}_{2} 155$. Since there are about $2^{156}$ isomorphism classes of elliptic curves defined over $\mathbb{F}_{2}{ }^{155}$, however, the probability that the GHS attack is applicable to a randomly chosen one is negligible. A further very detailed analysis for many other fields was carried out by Maurer, Menezes and Teske [19]. They identified all extension fields $\mathbb{F}_{2^{n}}$, where $160 \leqslant n \leqslant 600$, for which there should exist a cryptographically interesting elliptic curve over $\mathbb{F}_{2^{n}}$ such that the GHS attack is more efficient for that curve than for any other cryptographically interesting elliptic curve over $\mathbb{F}_{2^{n}}$. Ciet, Quisquater and Sica [5] discussed the security of fields of the form $\mathbb{F}_{2^{2 d}}$ where $d$ is a Sophie-Germain prime.

Galbraith, Hess and Smart [11] extended the GHS attack to isogeny classes of elliptic curves. The basic idea is to check whether a given elliptic curve is isogenous to an elliptic curve for which the basic GHS attack is effective. Then one computes the isogeny and reduces the ECDLP to that curve. This greatly increased the number of elliptic curves that succumb to the GHS attack for certain parameters.

The GHS attack has also been generalised to hyperelliptic curves, in even characteristic by Galbraith [9], and in odd characteristic by Diem [6]. Thériault considers, in [26], a special class of Artin-Schreier curves in any characteristic.

In this paper, we extend the GHS attack for elliptic curves in characteristic two even further, thereby considerably increasing the number of curves for which the basic GHS attack of [13] is applicable. In order to do so, we generalise the construction of [13] and [9] to arbitrary Artin-Schreier extensions, and this enables us to utilise different Artin-Schreier equations from those that had previously been considered. These new results are then combined with the technique of [11].

For example, for the field extension $\mathbb{F}_{2^{155}} / \mathbb{F}_{2^{5}}$, among the $2^{156}$ isomorphism classes of curves there are around $2^{104}$ that are vulnerable to attack under the extended method of [11]. Using the new construction, we find that around $2^{123}$ additional isomorphism classes should now be attackable.

On the other hand, it should be noted that the curves produced by our generalised construction, although they have the same genera as in [13], are no longer hyperelliptic. As a consequence, solving the discrete logarithm problem in the divisor class group of these curves is much more complicated, and is in general slower by a factor polynomial in the genus. The precise efficiency and practical implications have yet to be determined.

In the paper we further give a formula for the characteristic polynomial of Frobenius of the curves constructed using our method, and we discuss the conditions under which the discrete logarithm problem is preserved when mapped to the corresponding divisor class group by the norm-conorm homomorphism. Similar statements for the norm-conorm homomorphism have been obtained by Diem [6]. We additionally discuss a number of other possible variations of the construction, and conclude that they are unlikely to yield any further improvements. We also address the algorithmic issues of computing the final curves and solving the discrete logarithm on them.

The results of this paper show that curves defined over fields of composite extension degree over $\mathbb{F}_{2}$, especially 155 , may possibly be more susceptible to Weil descent attacks than is suggested by previous methods. Our techniques do not, however, pose a threat for prime extension degrees in small characteristic, or prime fields in large characteristic.

The remainder of the paper is organised as follows. In Section 2 we describe the general setting that is considered throughout the paper. In Section 3 we provide statements on Artin-Schreier extensions and base automorphisms. In Section 4 we explain the general Weil descent construction for Artin-Schreier extensions, and we make statements about its 
main invariants - such as its genus, the kernel of the norm-conorm homomorphism, and the characteristic polynomial of Frobenius. In Section 5 we specialise to the case of even characteristic and elliptic curves, and we generalise the original construction of [13].

In Section 6 we are ready to apply the theory developed in the preceding sections, to investigate alternative efficient constructions that can be carried out in the elliptic curve case in even characteristic. In Section 7 we briefly address algorithmic issues of computing the final curves and solving the discrete logarithm. In Section 8 we investigate various possibly more effective extensions and variations, and in Sections 9 and 10 we provide general statements on the norm-conorm homomorphism and the characteristic polynomial of Frobenius, which are used in the earlier sections. Section 11 finally contains the conclusion.

\section{Mapping the discrete logarithm problem}

Let $E$ be a function field of transcendence degree one over the finite exact constant field $K$, let $C / E$ be a finite extension, and let $U_{1}$ be a finite subgroup of $\operatorname{Aut}(C)$. The fixed field of $U_{1}$ in $C$ is denoted by $C^{U_{1}}$. We are mainly interested in the case where $E$ is the function field of an elliptic curve.

We obtain a homomorphism of the divisor class groups $\phi: \mathcal{C l}(E) \longrightarrow \mathcal{C l}\left(C^{U_{1}}\right)$ by

$$
\mathrm{N}_{C / C} U_{1} \circ \operatorname{Con}_{C / E},
$$

the conorm from $E$ to $C$ followed by the norm from $C$ to $C^{U_{1}}$. The divisor class groups of degree-zero divisors are denoted by $\mathrm{Cl} l^{0}(E)$ and $C l^{0}\left(C^{U_{1}}\right)$. There are two main objectives: first, the norm-conorm homomorphism $\phi$ should map a given discrete logarithm problem in $C l^{0}(E)$ sufficiently faithfully to $C l^{0}\left(C^{U_{1}}\right)$; second, subject to the first condition, the genus and the constant field of $C^{U_{1}}$ should be as small as possible.

The next four sections describe how such $C$ and $U_{1}$, not necessarily optimal in the above sense, can be constructed from $E$ in terms of Artin-Schreier extensions. We also give statements about the kernel of $\phi$, the $L$-polynomial of $C^{U_{1}}$ and its genus, based on general theorems that are proved later, in Sections 9 and 10.

\section{Artin-Schreier extensions with base automorphism}

In this section we describe methods that lead to the Weil descent techniques for ArtinSchreier extensions, as used in the next few sections, generalising those of [6] and [13]. For the following theory about Artin-Schreier extensions, see [2, pp. 22-24], [22, pp. 275-281] and [25, p. 115].

Let $F / K$ be an algebraic function field of characteristic $p$ and transcendence degree one over the exact constant field $K$. Let $\wp(x)=x^{p}-x$ be the Artin-Schreier operator. We have a 1-1 correspondence of $\mathbb{F}_{p}$-modules $\Delta \leqslant F^{+}$with $\wp(F) \subseteq \Delta$ and abelian extensions of $F$ of exponent $p$ within a fixed separable closure $\bar{F}$ of $F$, given by

$$
\Delta \mapsto C=F\left(\wp^{-1}(\Delta)\right)
$$

If $\Delta$ has finite dimension $m$, then $[C: F]=p^{m}$. Furthermore, there is a non-degenerate bilinear form

$$
\langle\cdot, \cdot\rangle: G(C / F) \times \Delta / \wp(F) \longrightarrow \mathbb{F}_{p}
$$

given by $\langle\tau, f\rangle=y \tau-y$, where $y \in \wp^{-1}(f)$ and $y \tau=\tau^{-1} y=\tau^{-1}(y)$. 
Let $\sigma \in \operatorname{Aut}(F)$ be an automorphism of finite order $n$, let $U=\langle\sigma\rangle$ be the cyclic group generated by $\sigma$, and assume from now on that $\Delta$ is $\sigma$-invariant: that is, an (additive) $\mathbb{F}_{p}[\sigma]$-module. The extension $C / F^{U}$ is then Galois of degree $p^{m} n$ with exact sequence

$$
1 \longrightarrow G(C / F) \longrightarrow G\left(C / F^{U}\right) \longrightarrow G\left(F / F^{U}\right) \longrightarrow 1 .
$$

This means that $G\left(C / F^{U}\right)$ is of exponent $n p$, and that $\sigma$ can be extended to an automorphism of $C$ of order $n$ or $n p$, denoted by $\sigma_{1}$. We let $U_{1}=\left\langle\sigma_{1}\right\rangle$. The sequence is split if and only if $G\left(C / F^{U}\right)$ contains an extension of $\sigma$ of order $n$, and a sufficient condition for this is that $p \nmid n$. In other words, if $\sigma_{1}$ is an extension of order $n p$, then $\sigma_{1}^{p \lambda}$ is an extension of order $n$, where $p \lambda \equiv 1 \bmod n$.

If $\Delta^{\prime} \subseteq \Delta$ is an $\mathbb{F}_{p}$-submodule of $\Delta$ and $\tau \in G\left(C / F^{U}\right)$, we have $\tau F\left(\wp^{-1}\left(\Delta^{\prime}\right)\right)=$ $F\left(\wp^{-1}\left(\tau \Delta^{\prime}\right)\right)$; consequently,

$$
G\left(C / F\left(\wp^{-1}\left(\tau \Delta^{\prime}\right)\right)\right)=\tau G\left(C / F\left(\wp^{-1}\left(\Delta^{\prime}\right)\right)\right) \tau^{-1} .
$$

The group $G(C / F)$ can be viewed as a right $\mathbb{F}_{p}\left[\sigma_{1}\right]$-module via conjugation, $\tau^{\sigma_{1}}=\sigma_{1} \tau \sigma_{1}^{-1}$ for $\tau \in G(C / F)$, and $\Delta / \wp(F)$ can be viewed as a right $\mathbb{F}_{p}\left[\sigma_{1}\right]$-module (or $\mathbb{F}_{p}[\sigma]$-module) by $f \sigma_{1}=f \sigma=\sigma^{-1}(f)$. Under these conditions, the pairing $\langle\cdot, \cdot\rangle$ in (1) is $\mathbb{F}_{p}\left[\sigma_{1}\right]$-linear:

$$
\left\langle\tau^{\sigma_{1}}, f\right\rangle=\left\langle\tau, f \sigma_{1}\right\rangle=\langle\tau, f \sigma\rangle .
$$

By duality, the pairing $\langle\cdot, \cdot\rangle$ leads to an $\mathbb{F}_{p}\left[\sigma_{1}\right]$-module isomorphism of $G(C / F)$ and $\Delta / \wp(F)$.

The following theorem gives precise conditions under which (2) is split in the case where $\Delta$ is a cyclic $\mathbb{F}_{p}[\sigma]$-module. For the general case, one can decompose $\Delta$ into a direct sum of cyclic factors and then apply the theorem.

THEOREM 3. Let $f \in \Delta$, and assume that $\Delta / \wp(F)$ is the cyclic $\mathbb{F}_{p}[\sigma]$-module generated by f. Let $m_{f} \in \mathbb{F}_{p}[t]$ be the monic polynomial of smallest degree such that $f_{m_{f}}(\sigma) \in \wp(F)$, and let $c_{f}=\left(t^{n}-1\right) / m_{f} \in \mathbb{F}_{p}[t]$.

(i) Any extension of $\sigma$ has order $n$ or $n p$; the exponent of $G\left(C / F^{U}\right)$ is $n p$.

(ii) There are extensions of $\sigma$ of order $n$ and $n p$ if and only if $f\left(\sigma^{n}-1\right) /(\sigma-1) \notin \wp(F)$. In this case, if $\sigma_{1}$ is an extension of order $n$, then $\sigma_{1} \tau$ is an extension of order $n p$ for every $\tau \in G(C / F)$ with $\left\langle\tau, f\left(\sigma^{n}-1\right) /(\sigma-1)\right\rangle \neq 0$. Conversely, if $\sigma_{1}$ has order $n p$, then there is $\tau \in G(C / F)$ with $\left\langle\tau, f\left(\sigma^{n}-1\right) /(\sigma-1)\right\rangle \neq 0$ such that $\sigma_{1} \tau$ has order $n$.

(iii) If $f\left(\sigma^{n}-1\right) /(\sigma-1) \in \wp(F)$, then the extensions of $\sigma$ have order $n$ if and only if $v c_{f}(\sigma)=0$ where $v \in \wp^{-1}\left(f m_{f}(\sigma)\right)$, and order $n p$ otherwise.

The conditions $f\left(\sigma^{n}-1\right) /(\sigma-1) \in \wp(F), c_{f}(1)=0$ and $v_{t-1}\left(m_{f}\right) \neq p^{v_{p}(n)}$ are equivalent, and $p \nmid n$ implies that $f\left(\sigma^{n}-1\right) /(\sigma-1) \notin \wp(F)$ or $v c_{f}(\sigma)=0$.

Proof. Since $\sigma$ has order $n$, we have $m_{f} \mid\left(t^{n}-1\right)$ and $c_{f} \in \mathbb{F}_{p}[t]$. Statement (i) follows from sequence (2) and its exactness.

We prove statement (ii). If $\sigma_{1}$ is an extension of $\sigma$, then every other extension can be written in the form $\sigma_{1} \tau$ with $\tau \in G(C / F)$, because (2) is exact. We have

$$
\left(\sigma_{1} \tau\right)^{n}=\tau^{\left(\sigma_{1}^{n}-1\right) /\left(\sigma_{1}-1\right)} \sigma_{1}^{n}
$$

by a straightforward calculation, and $\tau^{\sigma_{1}^{n}}=\tau$ because $\sigma_{1}^{n} \in G(C / F)$ by (2). If there are extensions of order $n$ and $n p$, then there exists $\tau$ such that $\sigma_{1}$ has order $n$ and $\sigma_{1} \tau$ has order $n p$, and hence

$$
\tau^{\left(\sigma_{1}^{n}-1\right) /\left(\sigma_{1}-1\right)} \neq 1
$$


Then

$$
\left\langle\tau^{\left(\sigma_{1}^{n}-1\right) /\left(\sigma_{1}-1\right)}, f\right\rangle \neq 0
$$

since otherwise, as $\tau^{\left(\sigma_{1}^{n}-1\right) /\left(\sigma_{1}-1\right)}$ is $\sigma_{1}$-invariant,

$$
\left\langle\tau^{\left(\sigma_{1}^{n}-1\right) /\left(\sigma_{1}-1\right)}, f \sigma^{i}\right\rangle=0, \quad \text { for } 1 \leqslant i \leqslant n
$$

and then $\left\langle\tau^{\left(\sigma_{1}^{n}-1\right) /\left(\sigma_{1}-1\right)}, w\right\rangle=0$ for all $w \in \Delta / \wp(F)$, which is impossible because $\langle\cdot, \cdot\rangle$ is non-degenerate. We have

$$
\left\langle\tau^{\left(\sigma_{1}^{n}-1\right) /\left(\sigma_{1}-1\right)}, f\right\rangle=\left\langle\tau, f\left(\sigma^{n}-1\right) /(\sigma-1)\right\rangle \neq 0,
$$

and thus

$$
f\left(\sigma^{n}-1\right) /(\sigma-1) \notin \wp(F) .
$$

Conversely, assume that $f\left(\sigma^{n}-1\right) /(\sigma-1) \notin \wp(F)$ holds true. If $\sigma_{1}$ has order $n$, we choose any $\tau$ with $\left\langle\tau, f\left(\sigma^{n}-1\right) /(\sigma-1)\right\rangle \neq 0$. Then

$$
\tau^{\left(\sigma_{1}^{n}-1\right) /\left(\sigma_{1}-1\right)} \neq 1
$$

and $\sigma_{1} \tau$ has order $n p$ by (4). If $\sigma_{1}$ has order $n p$, then $\tau_{1}=\sigma_{1}^{n} \in G(C / F)$ by (2), $\tau_{1} \neq 1$ and $\tau_{1}$ is $\sigma_{1}$-invariant. This implies that $\left\langle\tau_{1}, f\right\rangle \neq 0$, using an analogous reasoning process to that used above. We can find $\tau$ with $\left\langle\tau, f\left(\sigma^{n}-1\right) /(\sigma-1)\right\rangle=-\left\langle\tau_{1}, f\right\rangle$, and hence

$$
\left\langle\tau^{\left(\sigma_{1}^{n}-1\right) /\left(\sigma_{1}-1\right)} \tau_{1}, f\right\rangle=0 \text {. }
$$

Now $\tau^{\left(\sigma_{1}^{n}-1\right) /\left(\sigma_{1}-1\right)} \tau_{1}$ is $\sigma_{1}$-invariant, and we see that $\left\langle\tau^{\left(\sigma_{1}^{n}-1\right) /\left(\sigma_{1}-1\right)} \tau_{1}, w\right\rangle=0$ for all $w \in \Delta / \wp(F)$. Hence

$$
\tau^{\left(\sigma_{1}^{n}-1\right) /\left(\sigma_{1}-1\right)} \tau_{1}=\tau^{\left(\sigma_{1}^{n}-1\right) /\left(\sigma_{1}-1\right)} \sigma_{1}^{n}=1
$$

and $\sigma_{1} \tau$ has order $n$ by (4). This proves statement (ii).

We proceed to prove statement (iii) and the last statement. As in [13], we let $v \in$ $\wp^{-1}\left(f m_{f}(\sigma)\right) \subseteq F$ and $y \in \wp^{-1}(f)$. Then $y m_{f}\left(\sigma_{1}\right)=v+\lambda$ for some $\lambda \in \mathbb{F}_{p}$, and $\sigma_{1}$ has order $n$ if and only if

$$
y\left(\sigma_{1}^{n}-1\right)=y m_{f}\left(\sigma_{1}\right) c_{f}\left(\sigma_{1}\right)=v c_{f}(\sigma)+\lambda c_{f}(1)=0 .
$$

Note that $v c_{f}(\sigma) \in \mathbb{F}_{p}$ since $\wp\left(v c_{f}(\sigma)\right)=\wp(y) m_{f}(\sigma) c_{f}(\sigma)=f\left(\sigma^{n}-1\right)=0$. As in [13], we see that for every $\lambda \in \mathbb{F}_{p}$ there is an extension $\sigma_{1}$ with $y m_{f}\left(\sigma_{1}\right)=v+\lambda$. It follows that extensions of order $n$ and $n p$ exist if and only if $c_{f}(1) \neq 0$. Thus $c_{f}(1)=0$ is equivalent to $f\left(\sigma^{n}-1\right) /(\sigma-1) \in \wp(F)$ by statement (ii), and it is obviously equivalent to $v_{t-1}\left(m_{f}\right) \neq p^{v_{p}(n)}$. If $c_{f}(1)=0$, then $\sigma_{1}$ has order $n$ precisely if $v c_{f}(\sigma)=0$, by (5). This proves statement (iii). Finally, if $p \nmid n$, then we have extensions of order $n$, so $f\left(\sigma^{n}-1\right) /(\sigma-1) \notin \wp(F)$ or $v c_{f}(\sigma)=0$ holds true by statements (ii) and (iii).

We remark that analogous results can be obtained for Kummer extensions.

\section{Weil descent with Artin-Schreier extensions}

We describe now how the discrete logarithm in the divisor class group of an ArtinSchreier extension of small genus over a large finite field can be related to an equivalent discrete logarithm problem in the divisor class group of a curve of larger genus but defined over a smaller finite field. 
Let

$$
q=p^{r}, \quad k=\mathbb{F}_{q} \quad \text { and } \quad K=\mathbb{F}_{q^{n}} .
$$

The exact constant field of $F$ is assumed to be $K$. Let $\sigma$ be a Frobenius automorphism of $F$ with respect to $K / k$. By this we mean that $\sigma$ restricts to the Frobenius automorphism of $K / k$ and has order $[K: k]$ on $F$ such that $F / F^{U}$ with $U=\langle\sigma\rangle$ is a constant field extension of degree $n$. We could, for example, choose $F=K(x)$ and $\sigma$ to be the extension of the Frobenius automorphism of $K / k$ via $\sigma(x)=x$.

Let

$$
\begin{gathered}
\Delta=f \mathbb{F}_{p}[\sigma]+\wp(F), \\
C=F\left(\wp^{-1}(\Delta)\right), \\
E_{h}=F\left(\wp^{-1}(h)\right), \quad \text { for } h \in \Delta,
\end{gathered}
$$

and

$$
m=\operatorname{dim}_{\mathbb{F}_{p}} \Delta / \wp(F)=\operatorname{deg}\left(m_{f}\right) .
$$

The goal is to construct an extension $\sigma_{1}$ of $\sigma$ on $C$ which is a Frobenius automorphism of $C$ with respect to $K_{1} / k$ where $K_{1}$ is the exact constant field of $C$. We can then form $C^{U_{1}}$ where $U_{1}=\left\langle\sigma_{1}\right\rangle$, and map the discrete logarithm problem in $\mathrm{Cl}^{0}\left(E_{h}\right)$ to $\mathrm{Cl}^{0}\left(C^{U_{1}}\right)$ using

$$
\phi_{h}: \operatorname{Cl}\left(E_{h}\right) \longrightarrow \mathcal{C l}\left(C^{U_{1}}\right) \quad \text { defined by } \mathrm{N}_{C / C^{U_{1}}} \circ \operatorname{Con}_{C / E_{h}} \text {. }
$$

It will not always be the case that the discrete logarithm problem in $\mathrm{Cl} l^{0}\left(C^{U_{1}}\right)$ is equivalent to that in $\mathcal{C} l^{0}\left(E_{h}\right)$.

The extension $C / K$ either is regular or involves a constant field extension of degree $p$, since there are no two constant field extensions of degree $p$ that are linearly disjoint over $F$. We can thus distinguish two cases: $K_{1}=K$ and $\left[K_{1}: K\right]=p$. We have $F K_{1}=$ $F\left(\wp^{-1}(\Delta \cap K)\right)$, so $K_{1}=K$ if and only if $\Delta \cap K \subseteq \wp(F)$.

Case $K_{1}=K$. It suffices to find any extension of $\sigma$ of order $n$, and Theorem 3 describes how this can be achieved. If $v_{t-1}\left(m_{f}\right)=p^{v_{p}(n)}$, statement (ii) applies, and we can find such an extension. Otherwise, statement (iii) applies, and we can find an extension of order $n$ only if $v c_{f}(\sigma)=0$. This criterion is reformulated in Lemma 6. By Theorem 3, $p \nmid n$ or $m=n$ are sufficient conditions for the existence of an extension of $\sigma$ of order $n$.

Case $\left[K_{1}: K\right]=p . \quad$ The extension $F K_{1} / F^{U}$ is cyclic of order $n p$ and

$$
1 \longrightarrow G\left(C / F K_{1}\right) \longrightarrow G\left(C / F^{U}\right) \longrightarrow G\left(F K_{1} / F^{U}\right) \longrightarrow 1
$$

is exact. The Frobenius automorphism of $F K_{1} / F^{U}$ thus extends to $C$, and any such extension will have order $n p$ (see statement (iii) in Theorem 3).

Lemma 6. Let $P$ be a $\sigma$-invariant place of degree one of $F$, and let $\pi \in P$ be a $\sigma$-invariant uniformiser. Let $f=\sum_{i=v_{P}(f)}^{\infty} \lambda_{i} \pi^{i}$ be the $P$-adic expansion of $f$.

If $\left[K_{1}: K\right]=p$, then $\operatorname{Tr}_{K / \mathbb{F}_{p}}\left(\lambda_{0}\right) \neq 0$ and $v_{t-1}\left(m_{f}\right) \neq 0$, and $\sigma$ extends to a Frobenius automorphism of $C$ with respect to $K_{1} / k$.

If $K_{1}=K$, then $\sigma$ extends to a Frobenius automorphism of $C$ with respect to $K / k$ if and only if $\operatorname{Tr}_{K / \mathbb{F}_{p}}\left(\lambda_{0}\right)=0$ or $v_{t-1}\left(m_{f}\right)=p^{v_{p}(n)}$. 
Proof. Corresponding to $P$, we have an embedding $\phi: F \longrightarrow K((\pi))$; also, $\phi(x)=$ $\sum_{i=v_{P}(x)}^{\infty} \phi_{i}(x) \pi^{i}$, and $\sigma$ extends to an automorphism of $K((\pi))$ that operates on the coefficients and leaves $\pi$ fixed. We can extend the $\mathbb{F}_{p}[\sigma]$-module structure of $F$ to $K((\pi))$ accordingly, and $\phi_{0}: F \longrightarrow K$ will be $\mathbb{F}_{p}[\sigma]$-linear. For $t_{0}$ transcendental over $\mathbb{F}_{p}$, we let $t=t_{0}^{r}$ and $\sigma_{0}$ the Frobenius automorphism of $K / \mathbb{F}_{p}$ be such that $\mathbb{F}_{p}[t] \subseteq \mathbb{F}_{p}\left[t_{0}\right]$ and $\sigma=\sigma_{0}^{r}$.

If $\left[K_{1}: K\right]=p$, then there is $a \in f \mathbb{F}_{p}[\sigma] \cap K$ with $a \notin \wp(F)$. Since $a(\sigma-1)=0$, we must have $v_{t-1}\left(m_{f}\right) \neq 0$. Let $w$ be a normal basis element for $K / \mathbb{F}_{p}$. Then there is $h \in \mathbb{F}_{p}\left[t_{0}\right]$ such that $a=w h\left(\sigma_{0}\right)$. The conditions $a \notin \wp(F),\left(t_{0}-1\right) \nmid h$ and $\left(t_{0}^{n r}-1\right) \nmid$ $h\left(t_{0}^{n r}-1\right) /\left(t_{0}-1\right)$ are equivalent. Then

$$
\begin{aligned}
\operatorname{Tr}_{K / \mathbb{F}_{p}}(a) & =a\left(\sigma_{0}^{n r}-1\right) /\left(\sigma_{0}-1\right) \\
& =w h\left(\sigma_{0}\right)\left(\sigma_{0}^{n r}-1\right) /\left(\sigma_{0}-1\right),
\end{aligned}
$$

which is non-zero if and only if $\left(t_{0}^{n r}-1\right) \nmid h\left(t_{0}^{n r}-1\right) /\left(t_{0}-1\right)$. Thus $a \notin \wp(F)$ is equivalent to $\operatorname{Tr}_{K / \mathbb{F}_{p}}(a) \neq 0$. Now $a \in \lambda_{0} \mathbb{F}_{p}[\sigma]$, so that $\operatorname{Tr}_{K / \mathbb{F}_{p}}(a) \neq 0$ implies that $\operatorname{Tr}_{K / \mathbb{F}_{p}}\left(\lambda_{0}\right) \neq 0$. That the Frobenius automorphism extends follows from the discussion preceding Lemma 6.

In the case $K_{1}=K$, we prove the lemma as follows. By Theorem 3, the Frobenius automorphism extends if and only if $v_{t-1}\left(m_{f}\right)=p^{v_{p}(n)}$ or $v c_{f}(\sigma)=0$. Assume that $v_{t-1}\left(m_{f}\right) \neq p^{v_{p}(n)}$; hence $c_{f}(1)=0$. In the proof of Theorem 3 , it can be seen that $v c_{f}(\sigma) \in \mathbb{F}_{p}$; thus $\phi\left(v c_{f}(\sigma)\right) \in \mathbb{F}_{p}$ and $\phi_{i}\left(v c_{f}(\sigma)\right)=0$ for all $i \neq 0$. We find that $v c_{f}(\sigma)=0$ if and only if $\phi_{0}\left(v c_{f}(\sigma)\right)=\phi_{0}(v) c_{f}(\sigma)=0$. Now $c_{f}=\left(t_{0}-1\right) c_{f}^{\prime}$ for some $c_{f}^{\prime} \in \mathbb{F}_{p}\left[t_{0}\right]$ since $c_{f}(1)=0$, and $\phi_{0}(v)\left(\sigma_{0}-1\right)=\phi_{0}\left(v^{p}-v\right)=\phi_{0}(f) m_{f}(\sigma)$ since $v^{p}-v=f m_{f}(\sigma)$. We obtain

$$
\begin{aligned}
\phi_{0}(v) c_{f}(\sigma) & =\phi_{0}(v)\left(\sigma_{0}-1\right) c_{f}^{\prime}\left(\sigma_{0}\right) \\
& =\phi_{0}(f) m_{f}\left(\sigma_{0}^{r}\right) c_{f}^{\prime}\left(\sigma_{0}\right) \\
& =\phi_{0}(f)\left(\sigma_{0}^{n r}-1\right) /\left(\sigma_{0}-1\right) \\
& =\operatorname{Tr}_{K / \mathbb{F}_{p}}\left(\phi_{0}(f)\right),
\end{aligned}
$$

and thus $v c_{f}(\sigma)=0$ if and only if $\operatorname{Tr}_{K / \mathbb{F}_{p}}\left(\phi_{0}(f)\right)=0$.

TheOREM 7. Let $h \in \Delta$ and $V=\left\{\tau \in U_{1}: h \tau \in \lambda h+\wp(F)\right.$ for some $\left.\lambda \in \mathbb{F}_{p}\right\}$. Then

$$
\begin{aligned}
\mathrm{N}_{E_{h} /\left(E_{h}\right)^{V}}^{-1}(0) & \subseteq \operatorname{ker}\left(\phi_{h}\right) \\
& \subseteq \mathrm{N}_{E_{h} /\left(E_{h}\right)^{V}}^{-1}\left(\left[p^{m-1}\right]^{-1}\left(\operatorname{Con}_{\left(E_{h}\right)^{V} / F^{V}}\left(\mathcal{C} l^{0}\left(F^{V}\right)\right)\right)\right),
\end{aligned}
$$

where $\left[p^{m-1}\right]$ is the 'multiplication-by- $p^{m-1}$ ' map.

Proof. The fields $E_{h}$ and $\tau E_{h}=E_{\tau h}$ for $\tau \in U_{1}$ are either equal or linearly disjoint over $F$. The definition of $V$ implies that $V E_{h} \subseteq E_{h}$, and $E_{h}=\tau E_{h}$ if and only if $\tau \in V$. Furthermore, the kernel of the restriction map $U_{1} \longrightarrow \operatorname{Aut}(F)$ is zero. The conditions of Proposition 23 are therefore fulfilled. We obtain $\left(E_{h}\right)^{V} \cap \tau\left(E_{h}\right)^{V}=F^{V}$ for all $\tau \in U_{1}$ with $\tau \notin V$. Applying Theorems 18 and 24 with $\phi=\phi_{h}$ gives the result.

Assume that $\mathcal{C l} l^{0}\left(E_{h}\right)$ has a large prime factor greater than $p$, which is not present in $\mathrm{Cl}^{0}(F)$. Theorem 7 says that if $K_{1}=K$ and $V=\{1\}$, the large prime factor will not be mapped to zero under $\phi_{h}$. If $V \neq\{1\}$ and the large prime factor is not present in $e l^{0}\left(\left(E_{h}\right)^{V}\right)$, then it will be mapped to zero. If, on the other hand, $\left[K_{1}: K\right]=p$, we see 
that $\sigma_{1}^{n} \in G\left(E_{h} / F\right)$ has order $p$ and $G\left(E_{h} / F\right) \subseteq V$, and thus $\left(E_{h}\right)^{V} \subseteq F^{V}$. This means that the large prime factor will always be mapped to zero in this case.

We now state upper and lower bounds on the genus of $C$.

THEOREM 8. Let $f \in \Delta$, and assume that $\Delta / \wp(F)$ is the cyclic $\mathbb{F}_{p}[\sigma]$-module, of $\mathbb{F}_{p^{-}}$ dimension $m$, generated by $f$. Then $m \leqslant n$ and

$$
g_{C} \leqslant \begin{cases}\left(p^{m}-1\right) /(p-1)\left(g_{E_{f}}-g_{F}\right)+g_{F} & \text { if } f \text { has } \sigma \text {-invariant poles, } \\ \left(p^{m}-1\right) /(p-1)\left(n \cdot g_{E_{f}}-g_{F}\right)+g_{F} & \text { otherwise. }\end{cases}
$$

On the other hand, if $m \geqslant 2$ and $F\left(\wp^{-1}(f, f \sigma)\right)$ has a genus greater than $g_{E_{f}}$, then

$$
g_{C} \geqslant p^{m-2} g_{E_{f}} /\left[K_{1}: K\right]+1 \text {. }
$$

Proof. Since $\sigma$ has order $n$, we have at most $n$ different elements $f \sigma^{i}$, and because $\Delta$ is generated by $f$, it then follows that $m \leqslant n$.

Using the genus formula of [25, III.7.8] we see that $g_{E_{h}} \leqslant n g_{E_{f}}$ for any $h \in \Delta$ since $h$ is an $\mathbb{F}_{p}$-linear combination of the conjugates $f \sigma^{i}$, and the numbers $m_{\sigma^{i}(P)}$ for $h$ and any given $P$ are thus less than or equal to the maximum of the numbers $m_{\sigma^{i}(P)}$ for $f$. Also, every place has at most $n$ conjugate places $\sigma^{i}(P)$. If $f$ has only $\sigma$-invariant poles, then $g_{E_{h}} \leqslant g_{E_{f}}$, since the numbers $m_{P}$ for $h$ and any given $P$ are less than or equal to the numbers $m_{P}$ for $f$. The upper bounds then follow from Corollary 38 with $U_{1}=\{1\}$ and $G=H=G(C / F)$. Here, the subgroups $H_{v}$ of $H$ of index $p$ correspond to one-dimensional $\mathbb{F}_{p}$-vector spaces contained in $\Delta / \wp(F)$ via $H_{v}=G\left(C / E_{f_{v}}\right)$, where $f_{v}$ spans a non-trivial $\mathbb{F}_{p}$-vector space in $\Delta / \wp(F)$. There are $\left(p^{m}-1\right) /(p-1)$ many $f_{v}$ and $H_{v}$. All the constant field extension degrees occurring in Corollary 38 are 1 because $U_{1}=\{1\}$.

The lower bounds are obtained from the Riemann-Hurwitz genus formula, which gives $2 g_{C}-2 \geqslant\left(2\left(g_{E_{f}}+1\right)-2\right) p^{m-2} /\left[K_{1}: K\right]$.

The lower bound of Theorem 8 is not very sharp. However, the main point here is that it shows that the genus of $C$ is exponential in $m$.

If (2) is split, we can also apply Theorem 26 and compute the $L$-polynomial of $C^{U_{1}}$, using the notation $G=G\left(C / F^{U_{1}}\right)$ and $H=G(C / F)$. Here, as in the proof of Theorem 8, subgroups $H_{v}$ of $H$ of index $p$ correspond to one-dimensional $\mathbb{F}_{p}$-vector spaces contained in $\Delta / \wp(F)$ via $H_{v}=G\left(C / E_{f_{v}}\right)$, where $f_{v}$ spans a non-trivial $\mathbb{F}_{p}$-vector space in $\Delta / \wp(F)$. There are $\left(p^{m}-1\right) /(p-1)$ many $f_{v}$ and $H_{\nu}$. Furthermore,

$$
U_{1 v}=\left\{\tau \in U_{1}: f_{\nu} \tau \in \lambda f_{\nu}+\wp(F) \text { for some } \lambda \in \mathbb{F}_{p}\right\} \text {. }
$$

\section{Generalising the basic GHS attack}

In [13], an Artin-Schreier construction has been applied to the case where $E_{f}$ is the function field of an elliptic curve and $F$ is the rational function field, over a finite field in characteristic two. We now describe a generalisation of this construction, obtained by specialising and applying the techniques of the previous sections.

Let $p=2$ and $\Delta=f \mathbb{F}_{2}[\sigma]+\wp(F)$, where $f=\gamma / x+\alpha+\beta x$ for $\gamma, \alpha, \beta \in K$ and $\gamma \beta \neq 0$. Furthermore, let $F=K(x)$. We have

$$
\Delta / \wp(F) \cong \mathbb{F}_{2}[t] /\left(m_{f}\right)
$$

with $m_{f}$ as in Theorem 3, of degree $m$. 
More precisely, if $m_{\gamma}$ and $m_{\beta}$ are polynomials in $\mathbb{F}_{2}[t]$ of minimal degree such that $\gamma m_{\gamma}(\sigma)=0$ and $\beta m_{\beta}(\sigma)=0$, then

$$
m_{f}= \begin{cases}\operatorname{lcm}\left(m_{\gamma}, m_{\beta}\right), & \text { for } \alpha \in \wp(F), \\ \operatorname{lcm}\left(m_{\gamma}, m_{\beta}, t+1\right), & \text { otherwise. }\end{cases}
$$

We remark that $\alpha \in \wp(F)$ is equivalent to $\operatorname{Tr}_{K / \mathbb{F}_{2}}(\alpha)=0$.

THEOREM 11. The Frobenius automorphism $\sigma$ of $F$ with respect to $K / k$ extends to a Frobenius automorphism of $C$ with respect to $K / k$ if and only if at least one of the conditions

$$
\operatorname{Tr}_{K / \mathbb{F}_{2}}(\alpha)=0, \quad \operatorname{Tr}_{K / k}(\gamma) \neq 0 \quad \text { or } \quad \operatorname{Tr}_{K / k}(\beta) \neq 0
$$

holds.

Proof. We have $K_{1}=K$ if and only if $\alpha \operatorname{lcm}\left(m_{\gamma}, m_{\beta}\right)(\sigma) \in \wp(F)$, and this in turn is equivalent to saying that at least one of the conditions $(t+1) \mid m_{\gamma} m_{\beta}$ or $\operatorname{Tr}_{K / \mathbb{F}_{2}}(\alpha)=0$ holds, which can be seen to be similar to the proof of Lemma 6.

In the case $K_{1}=K$, the Frobenius automorphism $\sigma$ of $F$ extends to a Frobenius automorphism of $C$ with respect to $K / k$ if and only if $\operatorname{Tr}_{K} / \mathbb{F}_{2}(\alpha)=0$ or $v_{t+1}\left(m_{f}\right)=2^{v_{2}(n)}$, by Lemma 6 , using the place $x=0$ and uniformiser $x$. Thus there exists a Frobenius automorphism of $C$ with respect to $K / k$ if and only if $\operatorname{Tr}_{K / \mathbb{F}_{2}}(\alpha)=0$, or both conditions $(t+1) \mid m_{\gamma} m_{\beta}$ and $v_{t+1}\left(m_{f}\right)=2^{v_{2}(n)}$ hold.

Now, if $n$ is even, then $v_{t+1}\left(m_{f}\right)=2^{v_{2}(n)}$ implies that $v_{t+1}\left(m_{\gamma}\right)=2^{v_{2}(n)}$ or $v_{t+1}\left(m_{\beta}\right)=$ $2^{v_{2}(n)}$, because of the definition of $m_{f}$ and (10); conversely, $v_{t+1}\left(m_{\gamma}\right)=2^{v_{2}(n)}$ or $v_{t+1}\left(m_{\beta}\right)=2^{v_{2}(n)}$ implies that $(t+1) \mid m_{\gamma} m_{\beta}$ and $v_{t+1}\left(m_{f}\right)=2^{v_{2}(n)}$. If $n$ is odd, then $(t+1) \mid m_{\gamma} m_{\beta}$ is equivalent to saying that $v_{t+1}\left(m_{\gamma}\right)=2^{v_{2}(n)}$ or $v_{t+1}\left(m_{\beta}\right)=2^{v_{2}(n)}$ holds, and implies that $v_{t+1}\left(m_{f}\right)=2^{v_{2}(n)}$. This shows that the Frobenius automorphism extends if and only if $\operatorname{Tr}_{K} / \mathbb{F}_{2}(\alpha)=0, v_{t+1}\left(m_{\gamma}\right)=2^{v_{2}(n)}$ or $v_{t+1}\left(m_{\beta}\right)=2^{v_{2}(n)}$ hold.

Finally, $\operatorname{Tr}_{K / k}(\gamma)=\gamma\left(\sigma^{n}+1\right) /(\sigma+1)$, and $v_{t+1}\left(m_{\gamma}\right)=2^{v_{2}(n)}$ is equivalent to $\gamma\left(\sigma^{n}+1\right) /(\sigma+1) \neq 0$. This proves the theorem.

If $\sigma_{1}$ is a Frobenius automorphism of $C$, then the genus of $C^{U_{1}}$ equals the genus of $C$, and Theorem 8 yields appropriate bounds. For the special choice of $f$ it is, however, possible to determine the genus precisely. The following result can be applied whenever the conditions in Theorem 11 are fulfilled.

TheOREM 12. Assume that $K_{1}=K$. The genus of $C=F\left(\wp^{-1}(\Delta)\right)$ is given by

$$
g_{C}=2^{m}-2^{m-\operatorname{deg}\left(m_{\gamma}\right)}-2^{m-\operatorname{deg}\left(m_{\beta}\right)}+1 .
$$

Proof. One-dimensional $\mathbb{F}_{2}$-subspaces of $\Delta / \wp(F)$ are given by non-zero elements in $\mathbb{F}_{2}[t] /\left(m_{f}\right)$ under the isomorphism (9). Let $v \in \Delta$ correspond to $w \in \mathbb{F}_{2}[t]$ of degree less than $m$. Then $v=f w(\sigma)$ and $E_{v}$ has genus 0 if $\gamma w(\sigma)=0$ or $\beta w(\sigma)=0$, and genus 1 otherwise. Thus for genus 0 we know that $w$ is divisible by $m_{\gamma}$ or by $m_{\beta}$. If $\alpha \in \wp(F)$, then $m_{f}=\operatorname{lcm}\left(m_{\gamma}, m_{\beta}\right)$. If $\alpha \notin \wp(F)$, then $(t+1) \mid m_{\gamma} m_{\beta}$, because we have assumed that $K_{1}=K$, which implies that $\alpha m_{\gamma}(\sigma) m_{\beta}(\sigma) \in \wp(F)$, and hence again that $m_{f}=\operatorname{lcm}\left(m_{\gamma}, m_{\beta}\right)$. The two cases $m_{\gamma} \mid w$ and $m_{\beta} \mid w$ thus lead to disjoint sets of non-zero $w$, since no non-zero $w \in \mathbb{F}_{2}[t]$ with $\operatorname{deg}(w)<m$ can be divisible by both $m_{\gamma}$ and $m_{\beta}$, as this would imply that $w$ is divisible by $m_{f}=\operatorname{lcm}\left(m_{\gamma}, m_{\beta}\right)$ and hence that $\operatorname{deg}(w) \geqslant m$. There are thus precisely $\left(2^{m-\operatorname{deg}\left(m_{\gamma}\right)}-1\right)+\left(2^{m-\operatorname{deg}\left(m_{\beta}\right)}-1\right)$ non-zero polynomials $w \in \mathbb{F}_{2}[t]$, of degree less than $m$, which are divisible by $m_{\gamma}$ or by $m_{\beta}$. 
Since there are $2^{m}-1$ non-zero polynomials $w \in \mathbb{F}_{2}[t]$ of degree less than $m$, we obtain the number of one-dimensional $\mathbb{F}_{2}$-subspaces of $\Delta / \wp(F)$ such that the associated degree-2 extensions have genus 1 , by subtracting the number of genus- 0 cases from all the cases; that is, $2^{m}-1-\left(2^{m-\operatorname{deg}\left(m_{\gamma}\right)}-1\right)-\left(2^{m-\operatorname{deg}\left(m_{\beta}\right)}-1\right)$. Using Corollary 38 and the remarks at the end of Section 4 , we finally obtain the result.

We want to link the results obtained so far to the results of [13]. Let $\gamma \in k$. Then $m_{\gamma}=t+1$ and $\Delta \cap K \subseteq K(\sigma+1) \subseteq \wp(F)$, so that $K_{1}=K$ and $C / K$ is regular. For the existence of the Frobenius automorphism with respect to $K / k$, we note that $\operatorname{Tr}_{K / k}(\gamma) \equiv$ $n$ mod 2 holds, and that $\operatorname{Tr}_{K / k}(\beta) \neq 0$ is equivalent to $(t+1)^{u} \mid m_{\beta}$, where $u=2^{v_{2}(n)}$. This shows that [19, Lemma 6, condition (2)] is necessary and sufficient, and that [13, condition $(\dagger)]$ is sufficient for the existence of the Frobenius automorphism. The genus of $C$ is equal to $2^{m-1}-2^{m-\operatorname{deg}\left(m_{\beta}\right)}+1$. Depending on whether or not $(t+1) \mid m_{\beta}$, this gives $m-\operatorname{deg}\left(m_{\beta}\right)=0$ or $m-\operatorname{deg}\left(m_{\beta}\right)=1$, and hence a genus of $2^{m-1}$ or $2^{m-1}-1$. Finally, similarly to the proof of Theorem 12 , we see that $F\left(\wp^{-1}(\Delta(\sigma+1))\right)$ has genus 0 and index 2 in $C$; hence $C$ is hyperelliptic. This recovers the main results about the construction in [13]. In addition, we now obtain the following, more precise, statement.

Corollary 13. Let $\gamma \in k$. The genus of $C$ is $2^{m-1}-1$ if and only if $\operatorname{Tr}_{K / \mathbb{F}_{q^{u}}}(\beta)=0$, where $u=2^{v_{2}(n)}$.

Proof. We have to prove that $(t+1) \nmid m_{\beta}$ is equivalent to $\operatorname{Tr}_{K / \mathbb{F}_{q^{u}}}(\beta)=0$. We can write $\beta=w h(\sigma)$ with $h \in k[t]$ and $w \in K$ a normal basis element over $k$ such that $K=w k[\sigma]$. Also, $v_{t+1}\left(t^{n}+1\right)=u$.

If the genus is $2^{m-1}-1$, then $(t+1) \nmid m_{\beta}$. Because $w\left(m_{\beta} h\right)(\sigma)=\beta m_{\beta}(\sigma)=0$ and $t^{n}+1$ is the $k[t]$-minimal polynomial of $w$, we find that $\left(t^{n}+1\right) \mid m_{\beta} h$ and $\left(t^{u}+1\right) \mid h$. Then $\beta=w\left(\sigma^{u}+1\right) h_{1}$ for some $h_{1} \in k[t]$ such that $h=\left(t^{u}+1\right) h_{1}$, and $\operatorname{Tr}_{K / \mathbb{F}_{q^{u}}}(\beta)=$ $\beta\left(\sigma^{n}+1\right) /\left(\sigma^{u}+1\right)=w\left(\sigma^{n}+1\right) h_{1}=0$.

Conversely, if $\operatorname{Tr}_{K / \mathbb{F}_{q^{u}}}(\beta)=\beta\left(\sigma^{n}+1\right) /\left(\sigma^{u}+1\right)=0$, then $m_{\beta} \mid\left(\left(t^{n}+1\right) /\left(t^{u}+1\right)\right)$ since $m_{\beta}$ is the $\mathbb{F}_{2}[t]$-minimal polynomial of $\beta$. But $\left(t^{n}+1\right) /\left(t^{u}+1\right)$ is coprime to $t+1$, so that $(t+1) \nmid m_{\beta}$. It follows that the genus is $2^{m-1}-1$.

For $h \in \Delta$ with $h=c / x+a+b x$, define $s(h)=\min \left\{s \geqslant 1: \sigma^{s}(c)=c\right.$ and $\left.\sigma^{s}(b)=b\right\}$. Then $\sigma^{s(h)}$ is the smallest power of $\sigma$ that maps the one-dimensional subspace of $\Delta / \wp(F)$ generated by $h$ to itself, or, in other words, such that $\sigma_{1}^{s(h)}$ yields an automorphism of $E_{h}$. For example, if $n / s(h)$ is odd, then $E_{h}=E_{\tilde{h}}$, where $\tilde{h}=c / x+\operatorname{Tr}_{K / \mathbb{F}_{q^{s(h)}}}(a)+b x \in \mathbb{F}_{q^{s(h)}}(x)$.

THEOREM 14. For the homomorphism $\phi_{h}: \mathcal{C l}\left(E_{h}\right) \longrightarrow \mathcal{C l}\left(C^{U_{1}}\right)$ given by the composition $\mathrm{N}_{C / C^{U_{1}}} \circ \operatorname{Con}_{C / E_{h}}$, we have

$$
\begin{aligned}
\mathrm{N}_{E_{h} /\left(E_{h}\right)^{\left\langle\sigma_{1}^{s(h)}\right\rangle}}^{-1}(0) & \subseteq \operatorname{ker}\left(\phi_{h}\right) \\
& \subseteq \mathrm{N}_{E_{h} /\left(E_{h}\right)^{\left\langle\sigma_{1}^{s(h)}\right\rangle}}^{-1}\left(\mathcal{C l}\left(\left(E_{h}\right)^{\left\langle\sigma_{1}^{s(h)}\right\rangle}\right)\left[2^{m-1}\right]\right) .
\end{aligned}
$$

Proof. This follows from Theorem 7 and $C l^{0}(F)=0$, since $F$ is rational.

Assume that $\sigma_{1}$ is a Frobenius automorphism of $C$ with respect to $K / k$, as in Theorem 11 . Theorem 14 then means that the discrete logarithm problem in $E_{h}$ will be preserved by $\phi_{h}$ in practical applications if and only if $s(h)=n$; that is, if $E_{h}$ does not come from a non-trivial 
constant field extension (is not defined by a subfield curve). Interestingly, $C^{U_{1}}$ is in a sense universal, in that it preserves discrete logarithms in large prime subgroups for all $E_{h}$ and $h \in \Delta$ such that $s(h)=n$.

The $L$-polynomial of $C^{U_{1}}$ or the characteristic polynomial of Frobenius over $k$ can be computed as follows.

THEOREM 15. Assume that $\sigma_{1}$ is a Frobenius automorphism of $C$ with respect to $K / k$. Let $S \subseteq f \mathbb{F}_{2}[\sigma]$ be a system of representatives under the operation of $U$ on $\Delta / \wp(F)$. Then

$$
L_{C^{U_{1}}}(t)=\prod_{h \in S} L_{\left(E_{h}\right)^{\left\langle\sigma_{1}^{s(h)}\right\rangle}}\left(t^{s(h)}\right) .
$$

Proof. We want to apply Theorem 26 using the remarks at the end of Section 4. Since $E_{\sigma h}=\sigma_{1}\left(E_{h}\right)$ and $G\left(C / \sigma_{1}\left(E_{h}\right)\right)=\sigma_{1} G\left(C / E_{h}\right) \sigma_{1}^{-1}$, a set of representatives $H_{v}$ under the operation of $U_{1}$ on subgroups of $G(C / F)$ of index 2 is given by

$$
\left\{G\left(C / E_{h}\right): h \in S, h \notin \wp(F)\right\} .
$$

Then

$$
U_{1 v}=\left\langle\sigma_{1}^{s(h)}\right\rangle \quad \text { and } \quad C^{H_{v} U_{1 v}}=\left(E_{h}\right)^{\left\langle\sigma_{1}^{s(h)}\right\rangle} \quad \text { for } v \text { corresponding to } h \in S .
$$

Let $k_{h}$ be the extension field of $k$ of degree $s(h)$. The constant field of $\left(E_{h}\right)^{\left\langle\sigma_{1}^{s(h)}\right\rangle}$ is equal to $k_{h}$. Furthermore,

$$
C^{G}=k(x), \quad C^{H U_{1 v}}=k_{h}(x) \quad \text { and } \quad\left(E_{h}\right)^{\left\langle\sigma_{1}^{s(h)}\right\rangle}=k(x) \quad \text { for } h \in \wp(F) .
$$

The result now follows from Theorem 26 , since $L$-polynomials of rational function fields are equal to 1 .

\section{Applications}

A representative for each isomorphism class of ordinary elliptic curves defined over $K$ with $p=2$ is given by $Y^{2}+X Y=X^{3}+\alpha X^{2}+\beta$, with $\beta \in K$ and $\alpha \in\{0, \omega\}$, where $\omega \in \mathbb{F}_{2^{u}}$ for $u=2^{v_{2}(n r)}$ is a fixed element with $\operatorname{Tr}_{\mathbb{F}_{2} u / \mathbb{F}_{2}}(\omega)=1$. The associated Artin-Schreier equation is $y^{2}+y=1 / x+\alpha+\beta^{1 / 2} x$, obtained by the transformation $Y=y / x+\beta^{1 / 2}, X=1 / x$ and multiplication by $x^{2}$. The same normalisation of $\alpha$ is also possible for the more general Artin-Schreier equations $y^{2}+y=\gamma / x+\alpha+\beta x$ of Section 5 .

It is the equation $y^{2}+y=1 / x+\alpha+\beta^{1 / 2} x$ that was used in [13] to perform the Weil descent. However, since $(a x+b) /(c x+d)$ for $a, b, c, d \in K$ with $a d-b c \neq 0$ is also a generator of $F$, we could make a substitution $x \mapsto(a x+b) /(c x+d)$ and apply the results of the previous sections to $f=(c x+d) /(a x+b)+\alpha+\beta^{1 / 2}(a x+b) /(c x+d)$. Since we aim at getting the smallest possible values of $m=\operatorname{deg}\left(m_{f}\right)$, because of Theorem 8 , we require $f$ to have $\sigma$-invariant poles. However, this implies that $b=\lambda a$ and $d=\mu c$ for $\lambda, \mu \in k$. Hence $(a x+b) /(c x+d)=(a / c)(x+\lambda) /(x+\mu)$. As $(x+\lambda) /(x+\mu)$ is $\sigma$-invariant, we can substitute $x$ for this. Writing $\gamma=a / c$, we obtain $f=1 /(\gamma x)+\alpha+\beta^{1 / 2} \gamma x$, and this is precisely of the form considered in Section 5. Similar reasoning holds if $a=0$ or $c=0$.

The question now is whether, for $\beta \in K$, there is a $\gamma \in K$ such that the polynomial $\operatorname{lcm}\left(m_{1 / \gamma}, m_{\beta^{1 / 2} \gamma}\right)$ has small degree in comparison with $n$. If we were to find such a $\gamma$, we could apply the results of Section 5 and reduce the discrete logarithm problem on the elliptic curve to that in the divisor class group of a higher-genus curve defined over $k$. The only general algorithm known thus far to find such a $\gamma$ works by computing all $\gamma$ such that $m_{1 / \gamma}$ has small degree, and then individually checking whether $m_{\beta^{1 / 2} \gamma}$ also has small degree. 
On the other hand, we can choose $\gamma_{1}, \gamma_{2} \in K$ such that $\operatorname{lcm}\left(m_{\gamma_{1}}, m_{\gamma_{2}}\right)$ has small degree in comparison with $n$, and define $\beta=\gamma_{1} \gamma_{2}$. Then $y^{2}+y=\gamma_{1} / x+\alpha+\gamma_{2} x$ and $Y^{2}+X Y=$ $X^{3}+\alpha X^{2}+\beta^{2}$ define isomorphic elliptic function fields. Heuristically, we would expect the map $\left(\gamma_{1}, \gamma_{2}\right) \mapsto \gamma_{1} \gamma_{2}$ to be almost injective for the $\gamma_{1}, \gamma_{2}$ under consideration, except for symmetry and scaling $\gamma_{1} \mapsto \lambda \gamma_{1}, \gamma_{2} \mapsto \lambda^{-1} \gamma_{2}$ by elements $\lambda \in k^{\times}$. This is also confirmed by examples. It follows that we (heuristically) considerably increase the number of elliptic curves that can be attacked by the basic GHS attack.

We now want to combine our results with the results of [11]. Assume, for simplicity, that $r$ and $n$ are odd, and that $n$ is prime so that $\alpha \in \mathbb{F}_{2}$ according to the above normalisation. Over $\mathbb{F}_{2}$, we have the factorisation into irreducible polynomials $t^{n}+1=(t+1) h_{1} \cdots h_{s}$ and $\operatorname{deg}\left(h_{i}\right)=d$ such that $n=s d+1$; see [20]. In this situation, the first non-trivial $m$ satisfies $d \leqslant m \leqslant d+1$, yielding $m_{f}=h_{i}$ or $m_{f}=(t+1) h_{i}$ by equation (10). Due to our generalisation we do not necessarily have $m=d+1$ as in $[11,13$, and in fact we are now concentrating on $m=d$. The number of Artin-Schreier equations as in Section 5 with $\alpha \in \mathbb{F}_{2}$ and $d \leqslant m \leqslant d+1$ is approximately equal to $2 s q^{2 d+2}$, whereas the number of equations among these with $m=d$ (which implies that $\alpha=0$ ) is approximately equal to $s q^{2 d}$. From these Artin-Schreier equations, we expect to obtain about $\min \left\{q^{n}, s q^{2 d-1} / 2\right\}$ associated elliptic curve equations, using the above transformations, and a system of representatives under the action of the 2-power Frobenius of cardinality $\min \left\{q^{n} /(n r), s q^{2 d-1} /(2 n r)\right\}$. Furthermore, as in [11], we expect these representatives to be distributed over the isogeny classes like arbitrary elliptic curves with $a=0$.

If $m=d$, we have $m_{f}=m_{\gamma}=m_{\beta},(t+1) \nmid m_{\gamma} m_{\beta}$ and $\alpha=0$. It follows that $\operatorname{Tr}_{K / k}(\gamma)=\operatorname{Tr}_{K / k}(\beta)=0$, and by the Theorems 11 and 14 the Weil descent technique does work because $\operatorname{Tr}_{K / \mathbb{F}_{2}}(\alpha)=0$ and $\gamma$ and $\beta$ are not in a subfield of $K$ since $n$ is prime. The resulting genus then satisfies $g_{C}=2^{d}-1$, by Theorem 12 . Note that in $[\mathbf{1 1}, \mathbf{1 3}]$ it is always the case that $m=d+1$ but $\operatorname{deg}\left(m_{\gamma}\right)=1$, so that the genus is of similar size, namely $2^{d}-1$ or $2^{d}$. Going back to the case $m=d$, we observe that if $\alpha=0$, then the group order of the elliptic curve is congruent to 0 modulo 4 , and if $\alpha=1$, then it is congruent to 2 modulo 4 (see [3, p. 38]). This means that curves with $\alpha=0$ represent half of all the (about $2 q^{n / 2}$ ) isogeny classes. Taking this into account, we discover from [11] that a proportion of $\min \left\{1, s q^{2 d-1} /\left(2 q^{n / 2} n r\right)\right\}$ of all elliptic curves over $K$ with $\alpha=0$ leads to curves of genus $2^{d}-1$ defined over $k$ with the equivalent discrete logarithm problem. Given a random elliptic curve with $\alpha=0$, we can find the associated elliptic curve (from which such a curve of genus $2^{d}-1$ can be computed) in running time $O(n \log (q) N)+O\left(q^{n / 4+\varepsilon}\right)$ and with probability $\min \left\{1, N / q^{n / 2}\right\}$, where $N \leqslant s q^{2 d-1} /(2 n r)$ and $\varepsilon>0$.

The case $n=31$ and $r=5$ is particularly interesting, since there is an IPsec curve [17] with $\alpha=0$, defined over $\mathbb{F}_{2155}$. This case has $d=5$ and $s=6$, and thus yields genus 31 ; these are feasible parameters, according to [18]. The heuristic probability that a random elliptic curve will give rise to a curve of genus 31 has been taken as approximately $2^{-52}$ with the method given in [11], whereas now we obtain

$$
s q^{2 d-1} /\left(2 q^{n / 2} n r\right) \approx 2^{-38} .
$$

The only algorithm known so far to find the elliptic curves from which the corresponding higher-genus curves are computed requires in the order of $s q^{2 d-1} /(2 n r) \approx 2^{39}$ many operations in $\mathbb{F}_{2^{155}}\left(q^{n / 4} \approx 2^{39}\right.$ here). This is not as efficient, but is still much faster than using the Pollard methods on the original curves. One can, however, additionally argue that the security of elliptic curves over $\mathbb{F}_{2155}$ does now at least partially depend on the difficulty of the problem of finding such higher-genus curves. 


\section{Algorithmic issues}

Thus far, our main objective has been to investigate whether there exist curves of sufficiently small genus, to whose divisor class group the discrete logarithm problem could be faithfully transferred. In this section we briefly discuss how to obtain explicit models for the resulting curves of Sections 4 and 5, and how to perform an index calculus method for solving the discrete logarithm problem. Note that the curves that we are considering are no longer necessarily hyperelliptic. Also, the most expensive step will be solving the discrete logarithm, and not the computation of the final curve and mapping the discrete logarithm.

\subsection{Explicit models and mapping the discrete logarithm}

We first exhibit an explicit model for $C$. Let $m=\operatorname{deg}\left(m_{f}\right)$. Note that the classes of $\sigma^{i}(f)$ for $0 \leqslant i \leqslant m-1$ form an $\mathbb{F}_{p}$-basis of $\Delta / \wp(F)$. It follows that $C$ is obtained by adjoining one root of every $y^{p}-y-\sigma^{i}(f)$ to $F$. In other words, $C=F\left[y_{0}, \ldots, y_{m-1}\right] / I$, where $I$ is the ideal of the polynomial ring $F\left[y_{0}, \ldots, y_{m-1}\right]$ generated by the polynomials $y_{i}^{p}-y_{i}-\sigma^{i}(f)$ for $0 \leqslant i \leqslant m-1$. We write $\bar{y}_{i}$ for the images of the $y_{i}$ in $C$, and we use the abbreviation $\bar{y}=\bar{y}_{0}$.

Assume that $\sigma$ extends to a Frobenius automorphism of $C$ with respect to $K / k$, again denoted by $\sigma$. After possibly replacing $y_{i}$ by $y_{i}+\mu_{i}$ for some $\mu_{i} \in \mathbb{F}_{p}$, we have

$$
\sigma\left(\bar{y}_{i}\right)=\bar{y}_{i+1}, \quad \text { for } 0 \leqslant i<m-1 .
$$

Also,

$$
\sigma\left(\bar{y}_{m-1}\right)=v-\sum_{i=0}^{m-1} \lambda_{i} \bar{y}_{i}
$$

holds, where the $\lambda_{i} \in \mathbb{F}_{p}$ are the coefficients of

$$
m_{f}=\sum_{i=0}^{m} \lambda_{i} t^{i},
$$

and $v \in F$ satisfies

$$
v^{p}-v=\sum_{i=0}^{m} \lambda_{i} \sigma^{i}(f) .
$$

Such $v$ will be determined up to the addition of an element in $\mathbb{F}_{p}$, and usually only one of the $p$ choices of $v$ will be the correct choice, so that $\sigma$ has order $n$ on $C$ (see the proof of Theorem 3). We obtain an explicit representation of the operation of $\sigma$ on $C$.

The field $C^{U_{1}}$ is the fixed field of $\sigma$ in $C$, and $F^{U}=k(x)$ is the fixed field of $\sigma$ in $F=K(x)$. Define

$$
\tilde{y}=\sum_{i=0}^{n-1} \sigma^{i}(\mu \bar{y})
$$

where $\mu$ is a normal basis element of $K$ over $\mathbb{F}_{p}$. Then $C^{U_{1}}=F^{U}(\tilde{y})$, because $\tilde{y} \in C^{U_{1}}$ and $C=F(\tilde{y})$, which in turn holds because $\tilde{y}$ has $[C: F]$ different conjugates under $G(C / F)$. To see the last statement, let $\tau \in G(C / F)$ and observe that $\sigma \tau \sigma^{-1} \in G(C / F)$. Define

$$
\lambda(\tau)=\tau(\bar{y})-\bar{y} \in \mathbb{F}_{p} .
$$

The map $\tau \mapsto\left(\lambda\left(\sigma^{-i} \tau \sigma^{i}\right)\right)_{0 \leqslant i \leqslant n-1}$ is injective because the right-hand-side values determine $\tau$ on all conjugates $\sigma^{i}(\bar{y})$. 
Then

$$
\begin{aligned}
\tau(\tilde{y}) & =\sum_{i=0}^{n-1} \tau \sigma^{i}(\mu \bar{y}) \\
& =\sum_{i=0}^{n-1} \sigma^{i}(\mu) \tau \sigma^{i}(\bar{y}) \\
& =\sum_{i=0}^{n-1} \sigma^{i}(\mu) \sigma^{i}\left(\sigma^{-i} \tau \sigma^{i}\right)(\bar{y}) \\
& =\sum_{i=0}^{n-1} \sigma^{i}(\mu) \sigma^{i}\left(\bar{y}+\lambda\left(\sigma^{-i} \tau \sigma^{i}\right)\right) \\
& =\sum_{i=0}^{n-1} \sigma^{i}(\mu) \sigma^{i}(\bar{y})+\sum_{i=0}^{n-1} \sigma^{i}(\mu) \lambda\left(\sigma^{-i} \tau \sigma^{i}\right) \\
& =\tilde{y}+\sum_{i=0}^{n-1} \sigma^{i}(\mu) \lambda\left(\sigma^{-i} \tau \sigma^{i}\right) .
\end{aligned}
$$

Since $\mu$ is a normal basis element, we can conclude that $\tilde{y}$ indeed has $[C: F]$ different conjugates. By computing the characteristic polynomial of $\tilde{y}$ over $F$ in $C$, we thus obtain a defining polynomial for $C^{U_{1}}$ in $F^{U}[t]$. The discrete logarithm can be mapped from $E_{f}$ to $C^{U_{1}}$, using the conorm map $\operatorname{Con}_{C / E_{f}}$ followed by the norm map $\mathrm{N}_{C / C^{U_{1}}}$. We give a very rough description of how this can be accomplished. It is best to work with suitable subrings (Dedekind domains) $R_{E_{f}}, R_{C}$ and $R_{C^{U_{1}}}$, and with ideals in these rings such that the ideal class groups are similar enough to the divisor class groups (preserving the large prime factor, for example). The conorm of a given ideal in $R_{E_{f}}$ then becomes the ideal generated in $R_{C}$ by the given ideal included in $R_{C}$. Using general techniques, we can compute a representation

$$
\bar{y}=h(\tilde{y}), \quad \text { with } h \in F[t] .
$$

For the norm ideal, we then form the product of the conjugated ideals in $R_{C}$ using $\sigma$. The substitution of $h(\tilde{y})$ for $\bar{y}$ and some further steps yield generators of the norm that are ideal in $R_{C^{U_{1}}}$.

\subsection{Index calculus}

Index calculus methods are employed for solving the discrete logarithm in the multiplicative group of finite fields or the divisor class group of hyperelliptic curves. They also apply to the divisor class group of general curves. We outline some of the main issues in our situation.

The basic observation is that every divisor class of $C^{U_{1}}$ of degree $g_{C^{U_{1}}}$ can be represented by an effective divisor of the same degree. Such a divisor decomposes uniquely into a sum of places of certain degrees and multiplicities, just as the case of rational integers and prime factorisations, and smoothness probabilities hold. These divisor class representatives can be computed by reduction techniques as described in [15], and this leads also to a mathod of computing in the divisor class group of $C^{U_{1}}$ that generalises the Cantor method for hyperelliptic curves. We remark that for hyperelliptic curves, addition takes $O\left(g_{C^{U_{1}}}^{2}\right)$ operations in $k$, whereas for a general $C^{U_{1}}$, addition takes $O\left(g_{C^{U_{1}}}^{4}\right)$ operations in $k$, and is hence considerably slower. 
The number of effective divisors of degree less than or equal to $g_{C^{U_{1}}}$ containing places of degree less than or equal to $d$ can usually be expressed as some explicit proportion of $q^{g_{C} U_{1}}$. For example, for $g_{C^{U_{1}}} \rightarrow \infty$ and $q$ fixed, we see that this number of smooth divisors is approximately at least

$$
q^{g^{U_{1}}} \exp \left(-\left(g_{C^{U_{1}}} / d\right) \log \left(g_{C^{U_{1}}} / d\right)\right), \quad \text { for } g_{C^{U_{1}}}^{c_{1}} \leqslant d \leqslant g_{C^{U_{1}}}^{c_{2}} \text { and } 0<c_{1}<c_{2}<1 \text { fixed. }
$$

From our formula for the characteristic polynomial of Frobenius of $C^{U_{1}}$ in Theorem 15, we see that

$$
g_{C^{U_{1}}}=\sum_{h \in S} s(h)
$$

by taking degrees, and then for the cardinality of the divisor class group

$$
\# C l^{0}\left(C^{U_{1}}\right)=q^{g} C^{U_{1}} \prod_{h \in S}\left(1+O\left(q^{-s(h) / 2}\right)\right)
$$

by evaluating at 1 . For every $h \in S$ we find that $s(h) \mid n$, and the number of $h \in S$ with $s(h) \mid s$ for given $s \mid n$ is less than or equal to $p^{s}$. If the number of divisors of $n$ is $O\left(\log \left(g_{C^{U_{1}}}\right)\right)$ and $q \geqslant p^{2}$, it follows that

$\# \mathcal{C} l^{0}\left(C^{U_{1}}\right)=\prod_{s \mid n} \prod_{s(h)=s}\left(1+O\left(p^{-s(h)}\right)\right)=O\left(q^{g} C^{U_{1}} g_{C^{U_{1}}}^{c}\right), \quad$ for some constant $c>1$,

and we expect this to be essentially true for $q=p$ because of possible alternating signs of the trace terms. If we divide the number of smooth divisors by the class number, it is hence reasonable to expect that a proportion of $\exp \left(-(1+o(1))\left(g_{C^{U_{1}}} / d\right) \log \left(g_{C^{U_{1}}} / d\right)\right)$ of all the divisor classes of degree $g_{C} U_{1}$ will be representable by a smooth divisor, thus leading to the usual smoothness probability. This would allow for a running time that is subexponential in $g_{C^{U_{1}}}$, with parameter $1 / 2$, for solving the discrete logarithm. For more details on computing discrete logarithms for general curves, see $[14,16]$.

\section{Further variations and observations}

It is of interest to see whether there are further variations or extensions of the GHS attack that would lead to smaller genera. In this section we investigate a number of such variations.

\subsection{Iterative descent}

Assume that $n=n_{1} n_{2}$. Instead of performing one descent from $K$ to $k$, we could consider descending first to $\mathbb{F}_{q^{n_{1}}}$, and then to $k$. The problem here is that $C^{U_{1}}$ is in general no longer an Artin-Schreier extension, so our techniques would not immediately apply. If, however, we start with an elliptic curve as in Section 6 and consider an associated Artin-Schreier model with $\gamma \in \mathbb{F}_{q^{n_{1}}}$, we do find that $C^{U_{1}}$ is hyperelliptic, or in other words that it is an Artin-Schreier extension. In this way, we obtain the following interesting result.

Assuming the generic cases, a descent from $K$ to $k$ leads to a hyperelliptic curve of genus of about $2^{n-1}$, whereas a descent from $K$ to $\mathbb{F}_{q^{n_{1}}}$ gives $2^{n_{1}-1}$. Using Theorem 8 , the descent from $\mathbb{F}_{q^{n_{1}}}$ to $k$ finally results in a curve of genus about

$$
\left(2^{n_{2}}-1\right) n_{2} 2^{n_{1}-1} \leqslant n_{2} 2^{n_{1}+n_{2}-1} .
$$

Thus, if $n_{1} \approx n_{2}$, this final curve has subexponential genus approximately $2^{(2+o(1)) \sqrt{n}}$, instead of exponential genus $2^{n}$. 
Let us look at the non-generic cases for $n=155, n_{1}=5$ and $n_{2}=31$. The smallest non-trivial descent from $\mathbb{F}_{2^{155}}$ to $\mathbb{F}_{2}$ leads to a genus of about $2^{20}$. On the other hand, there are descents from $\mathbb{F}_{2155}$ to $\mathbb{F}_{2^{5}}$ that result in genus $2^{5}-1$. Assuming the generic case $m=5$ for the descent from $\mathbb{F}_{2^{5}}$ to $\mathbb{F}_{2}$ then gives a genus at most $5\left(2^{5}-1\right)^{2}$.

While theoretically interesting, we do not expect these results to have any practical implications, because the resulting genera are still large.

\subsection{Descent from extensions}

If the descent from $\mathbb{F}_{q^{n}}$ to $\mathbb{F}_{q}$ does not yield a small enough genus, one could apply a change of variable to obtain a defining equation of $E_{f}$ defined over an extension field $\mathbb{F}_{\tilde{q}} \tilde{n}$ and descend to $\mathbb{F}_{\tilde{q}}$, thereby possibly yielding a smaller genus over another small base field for some suitable $\tilde{q}$ and $\tilde{n}$.

At least for prime $n$, however, this approach will give no improvement. To see this, we note that for any $n$, the degrees of the irreducible factors in $\mathbb{F}_{p}[t]$ of $t^{n}-1$ corresponding to primitive $n$th roots of unity are equal to the multiplicative order $m$ of $p$ modulo $n$. This $m$ is the smallest value of $\operatorname{deg}\left(m_{f}\right)$ that can occur for an elliptic curve over $\mathbb{F}_{q^{n}}$ that is not already defined over a subfield. For prime $n$, this $m$ is usually very big. Let $\tilde{m}$ be the multiplicative order of $p$ modulo $\tilde{n}$. The genus for a descent by $\tilde{n}$ is then approximately at least $p^{\tilde{m}}$. Thus, if $n \mid \tilde{n}$, then $\tilde{m} \geqslant m$ and the genus can only be bigger than before. Otherwise, if $n \nmid \tilde{n}$, then $n \mid\left[\mathbb{F}_{\tilde{q}}: \mathbb{F}_{p}\right]$ because $n$ is prime, and thus $\mathbb{F}_{\tilde{q}}$ is too big.

For composite $n$, improvements may be possible. Again, there are descents from $\mathbb{F}_{2^{155}}$ to $\mathbb{F}_{2}$ that yield genus approximately $2^{20}$, whereas the corresponding descents from $\mathbb{F}_{2}{ }^{155}$ to $\mathbb{F}_{2^{5}}$ yield genus about $2^{5}$ while $\mathbb{F}_{2^{5}}$ is still fairly small.

\subsection{Subfields and automorphisms}

A possible way of improving the construction in Sections 4 and 5 would be to consider subfields $L$ of $C^{U_{1}}$ and use $\phi_{f, L}=\mathrm{N}_{C^{U_{1} / L}} \circ \phi_{f}$ to map the discrete logarithm problem from $C l^{0}\left(E_{f}\right)$ to $e l^{0}(L)$. If the kernel of $\phi_{f, L}$ is small enough, this would lead to a very substantial improvement, because the genus of subfields is usually much smaller.

To approach this question, we first consider intermediate fields of the extension $C^{U_{1}} / F^{U_{1}}$. This extension is in general not Galois, and any intermediate field $L$ leads to an intermediate field $L K$ of $C / F$ with $\sigma_{1}(L K)=L K$. Thus $L K=F\left(\wp^{-1}\left(\Delta_{L}\right)\right)$ for a unique $\Delta_{L}$ with $\wp(F) \subseteq \Delta_{L} \subseteq \Delta$ and $\sigma\left(\Delta_{L}\right)=\Delta_{L}$. If $\Delta_{L} \neq \Delta$, then $f \notin \Delta_{L}$, and $E_{f}$ and $L K$ are thus linearly disjoint over $F$. Now $\mathrm{N}_{C / L K} \circ \operatorname{Con}_{C / E_{f}}=0$ by Lemma 16, and since $\mathrm{N}_{C^{U_{1} / L}} \circ \mathrm{N}_{C / C^{U_{1}}}=\mathrm{N}_{L K / L} \circ \mathrm{N}_{C / L K}$, we obtain $\phi_{f, L}=0$. Thus $\phi_{f, L}$ and intermediate fields of $C^{U_{1}} / F^{U_{1}}$ are of no use. We could still search for other subfields $L$ of $C^{U_{1}}$ that do not contain $F^{U_{1}}$ and yield a small kernel of $\phi_{f, L}$. One way of obtaining such subfields could be via the fixed fields of automorphism groups of $C$ containing the Frobenius automorphism. Indeed, if we had automorphisms $\rho \in \operatorname{Aut}(F / K)$ with $\rho(\Delta) \subseteq \Delta$, it should be possible to extend $\rho$ to $C$ in a similar way as was done with $\sigma$, under not too restrictive conditions. We have not found such automorphisms for $F=K(x)$ and $E_{f}$ defined by non-subfield curves. Even if no such automorphisms exist, there could still be useful subfields $L$, but this appears unlikely to happen, except perhaps in very rare cases.

Although automorphisms of $C^{U_{1}} / F^{U_{1}}$ may not be useful to find suitable subfields $L$ as shown above, they could be of use to speed up the discrete logarithm computation in $C^{U_{1}}$. We are given $2^{m}$ automorphisms in $G(C / F)$. For $\tau \in G(C / F)$ with $\tau \neq 1$, to restrict to an automorphism of $C^{U_{1}}$ we need $\tau \sigma_{1} \tau^{-1} \in U_{1}$. We have $\tau \sigma_{1} \tau^{-1}=\tau^{1-\sigma_{1}} \sigma_{1}$, and thus $\tau^{1-\sigma_{1}}=\tau \sigma_{1} \tau^{-1} \sigma_{1}^{-1} \in G(C / F) \cap U_{1}$. As $G(C / F) \cap U_{1}=\{1\}$, we obtain $\tau^{1-\sigma_{1}}=1$. 
Since $G(C / F)$ and $\Delta / \wp(F)$ are $\mathbb{F}_{p}\left[\sigma_{1}\right]$-isomorphic, it follows that if $m_{f}(1)=0$, then there is precisely one such $\tau$, and otherwise there is no such $\tau$. We remark that $\tau$ is the hyperelliptic involution in the case where $C^{U_{1}}$ is hyperelliptic. Thus $G\left(C^{U_{1}} / F^{U_{1}}\right)$ consists either of the identity only, or of the identity and the hyperelliptic involution. However, it is still possible that $C^{U_{1}}$ could have non-trivial automorphisms, obtained in a different way.

\subsection{Other composita}

The field composita in Sections 4 and 5 depend on the choice of the base field $F=K(x)$ within the function field $E_{f}$. We want to investigate what happens if other subfields (or none) are used, in the case of elliptic function fields $E_{f}$ in characteristic two.

If $K\left(x_{1}\right)$ and $K\left(x_{2}\right)$ are any two rational subfields of index 2 of the elliptic function field $E_{f}$, then there is an automorphism $\tau_{Q} \in \operatorname{Aut}\left(E_{f} / K\right)$ induced by a point translation map $P \mapsto P+Q$ such that $\tau_{Q}\left(K\left(x_{1}\right)\right)=K\left(x_{2}\right)$. In other words, we may assume that $x_{1}$ and $x_{2}$ are $x$-coordinates of Weierstrass models. Then $Q$ is the point where $x_{2}$ has its pole. We conclude that $E_{f} / K\left(x_{1}\right)$ and $E_{f} / K\left(x_{2}\right)$ are isomorphic, and hence it does not matter which rational subfield of index two is taken in Sections 4 and 5.

The methods of Sections 4 and 5 do not apply readily to other subfields of $E_{f}$. We make a few comments on what can be expected in terms of arbitrary field composita.

Elliptic subfields as common base fields $F$ are of no use. The extensions $E_{f} / F$ are abelian and unramified, so any compositum $C$ will be unramified over $F$ as well. This means, however, that $C$ has genus 1 and is again an elliptic function field. The corresponding elliptic curves are all isogenous. Should there be a Frobenius automorphism on $C$, then this would mean that the elliptic curve corresponding to $E_{f}$ is isogenous to an elliptic curve defined over the small finite field $k$. Other aspects of isogenous elliptic curves have been exploited in [11].

All other subfields $F$ must be rational, and of index at least 3, and such fields will indeed lead to alternative constructions. In order to estimate the resulting genus, we remark that the lower bound in Theorem 8 essentially remains valid in more general situations: as in Section 4, assume that we are given $C$ with a Frobenius automorphism $\sigma$ with respect to $K / k$ and an elliptic function field $E$ with $E \subseteq C$ such that $C=E(\sigma E) \cdots\left(\sigma^{m-1} E\right)$ for $m \leqslant n$ minimal. If $E(\sigma E)$ does not have genus at least 2, then it has genus 1 , and both $E(\sigma E) / E$ and $E(\sigma E) / \sigma E$ are unramified. This yields an unramified pyramid of fields. It follows that $C$ is unramified over $E$, and is hence elliptic, which reduces us to the uninteresting case discussed above. So we assume that $E(\sigma E)$ has genus at least 2 . Using the Riemann-Hurwitz genus formula, we find that the genus of $C$ is then bounded by $g_{C} \geqslant[C: E(\sigma E)]+1$ and $[C: E(\sigma E)] \geqslant 2^{m-2}$. If the fields $\sigma^{i} E$ are linearly disjoint over a common base field $F$ with $\sigma F \subseteq F$, we even have $[C: E(\sigma E)] \geqslant[E: F]^{m-2}$. The genus is thus exponential in $m$.

The main objective is hence again to minimise $m$ in comparison with $n$. A possible generalisation of the Artin-Schreier construction could be to use additive polynomials over a common rational base field $F$. This would lead to values of $m$ similar to those in Section 5, but could apply in more (or additional) cases. However, as $F$ would have index $2^{s}$ in $E$ for $s \geqslant 2$, the genus bound would be $g_{C} \geqslant 2^{s(m-2)}+1$, which is much larger than the construction of Section 5 .

Theoretically, there could also be completely different constructions of $C$, given $E$ and its conjugated fields. To be effective they would need to achieve a good 'compression' rate (that is, small values of $m$ ), because of the above lower bound for the genus. We do not know whether such constructions exist. 


\subsection{Characteristic three}

Weil descent with Artin-Schreier extensions as in Section 4 can also be carried out for elliptic curves in characteristic three. Here, Artin-Schreier equations that define elliptic curves have to be of the form $y^{3}-y=a x^{2}+b$ with $a, b \in K$. We thus expect to map the discrete logarithm problem to curves of genus $\Theta\left(3^{\operatorname{deg}\left(m_{f}\right)}\right)$ with $f=a y^{2}+b$. We remark that if $a=1$, we would again obtain an Artin-Schreier extension of degree 3.

Elliptic curves defined in this way are always supersingular and admit subexponential attacks via the MOV and FR reductions anyway $[\mathbf{8 , 2 1 ]}$ (with subexponential parameter $1 / 3$ instead of 1/2). We would expect these attacks to be more efficient than the GHS attack. Of course, analogous remarks hold for elliptic curves in even characteristic.

We remark that the main use of elliptic curves in characteristic three appears to be in identity-based cryptography [4]. For efficiency reasons, one usually considers supersingular curves. An alternative Weil descent construction for ordinary elliptic curves in characteristic three is described in [1].

\section{The kernel of the norm-conorm homomorphisms}

In this section, we prove the main results about the norm-conorm homomorphism that have been used in the proofs of Theorems 7 and 14 .

Lemma 16. Let $C / F$ be a finite extension of function fields, and let $E_{1}$ and $E_{2}$ be two intermediate function fields that are linearly disjoint over $F$. We have

$$
\mathrm{N}_{C / E_{2}}\left(\operatorname{Con}_{C / E_{1}}(x)\right)=\left[C: E_{1} E_{2}\right] \operatorname{Con}_{E_{2} / F}\left(\mathrm{~N}_{E_{1} / F}(x)\right)
$$

for all divisor classes $x \in \mathcal{C} l\left(E_{1}\right)$.

Proof. We have $\mathrm{N}_{C / E_{1} E_{2}}\left(\operatorname{Con}_{C / E_{1} E_{2}}(y)\right)=\left[C: E_{1} E_{2}\right] y$, so by the transitivity of the norm and conorm we can assume that $C=E_{1} E_{2}$. Furthermore, it suffices to prove the assertion for all but finitely many places $x=P$ of $E_{1}$. In other words, given any finite set of places of $E_{1}$, every divisor class in $E_{1}$ has a representing divisor whose support is disjoint from this set of places, by the approximation theorem.

Because $E_{1}$ and $E_{2}$ are linearly disjoint over $F$, we have $\left[E_{1} E_{2}: E_{1}\right]=\left[E_{2}: F\right]$ and $E_{1} \cap E_{2}=F$. Furthermore, for almost all places $P$ of $E_{1}$, the splitting behaviour of $P$ in $E_{1} E_{2}$ is the same as that of $P \cap F$ in $E_{2}$ (that is, their respective conorms $\operatorname{Con}_{E_{1} E_{2} / E_{1}}(P)$ and $\operatorname{Con}_{E_{2} / F}(P \cap F)$ consist of the same number of places, with the same relative degrees and ramification indices). If $P^{\prime}$ is any place of $E_{1} E_{2}$ above such a $P$, then by symmetry we have for the relative degrees,

$$
f\left(P^{\prime} / P^{\prime} \cap E_{2}\right)=f(P / P \cap F) .
$$

Since

$$
\mathrm{N}_{E_{1} E_{2} / E_{2}}\left(P^{\prime}\right)=f\left(P^{\prime} / P^{\prime} \cap E_{2}\right)\left(P^{\prime} \cap E_{2}\right)
$$

and

$$
\mathrm{N}_{E_{1} / F}(P)=f(P / P \cap F)(P \cap F),
$$

this gives

$$
\begin{aligned}
\mathrm{N}_{E_{1} E_{2} / E_{2}}\left(\operatorname{Con}_{E_{1} E_{2} / E_{1}}(P)\right) & =f(P / P \cap F) \operatorname{Con}_{E_{2} / F}(P \cap F) \\
& =\operatorname{Con}_{E_{2} / F}\left(\mathrm{~N}_{E_{1} / F}(P)\right) .
\end{aligned}
$$


The multiplication-by- $m$ map for $m \in \mathbb{Z}$ is denoted by $[m]$. In the following we use the notation and situation of Section 2, and view the norm and conorm maps as maps of the corresponding divisor class groups. Let $V$ denote a subgroup of $U_{1}$ such that $V E \subseteq E$; that is, $V$ restricts to a subgroup of $\operatorname{Aut}(E)$. Let $W$ denote the largest subgroup of $V$ such that $E^{W}=E$. We define

$$
\phi^{V}: \operatorname{el}\left(E^{V}\right) \longrightarrow \operatorname{el}\left(C^{U_{1}}\right)
$$

via the composition $\phi^{V}=\mathrm{N}_{C^{V} / C^{U_{1}}} \circ \operatorname{Con}_{C^{V} / E^{V}}$.

THEOREM 18. We have

$$
\phi=\phi^{V} \circ[\# W] \circ \mathrm{N}_{E / E^{V}}
$$

The kernel of $\phi$ thus consists at least of all elements contained in the kernel of the map $[\# W] \circ \mathrm{N}_{E / E^{V}}$.

Proof. The extensions $C^{W} / C^{V}$ and $E / E^{V}$ are Galois with group $V / W$, since $W$ is the kernel of the restriction map $V \longrightarrow \operatorname{Aut}(E)$, and is hence normal in $V$. The fields $E$ and $C^{V}$ are then linearly disjoint over $E^{V}$ because $E \cap C^{V}=E^{V},\left[E: E^{V}\right]=(V: W)$, $E C^{V}=C^{W}$ and $\left[E C^{V}: C^{V}\right]=\left[C^{W}: C^{V}\right]=(V: W)$ by the definition of $W$ and Galois theory. From Lemma 16, the transitivity of the norm and conorm maps and $\mathrm{N}_{C / C^{W}} \circ \operatorname{Con}_{C / C^{W}}=[\# W]$, we obtain

$$
\begin{aligned}
& \phi=\mathrm{N}_{C / C^{U_{1}}} \circ \operatorname{Con}_{C / E} \\
& =[\# W] \circ \mathrm{N}_{C^{W} / C^{U_{1}}} \circ \operatorname{Con}_{C^{W} / E}
\end{aligned}
$$

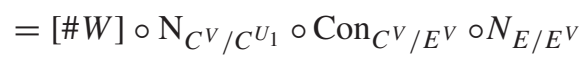

$$
\begin{aligned}
& =[\# W] \circ \phi^{V} \circ \mathrm{N}_{E / E^{V}} \\
& =\phi^{V} \circ[\# W] \circ \mathrm{N}_{E / E^{V}} \text {, }
\end{aligned}
$$

which proves (19). The statement about the kernel of $\phi$ is clear.

We remark that Theorem 18 can basically be applied recursively in the following way. Let $C_{V}$ be the normal closure of $C^{V}$ over $C^{U_{1}}$, and let

$$
\phi_{V}: \operatorname{el}\left(E^{V}\right) \longrightarrow \operatorname{el}\left(C^{U_{1}}\right)
$$

be defined by

$$
\phi_{V}=\mathrm{N}_{C_{V} / C^{U_{1}}} \circ \operatorname{Con}_{C_{V} / E^{V}} .
$$

Then $\phi_{V}=\left[\left[C_{V}: C^{V}\right]\right] \circ \phi^{V}$ and Theorem 18 can be applied to $\phi_{V}$ with $V$ replaced by any larger group $V^{\prime}$ such that $V^{\prime} E^{V} \subseteq E^{V}$.

Let $U_{1} / / V$ denote a set of coset representatives such that $U_{1}=\cup_{\sigma \in U_{1} / / V} \sigma V$ and $1 \in U_{1} / / V$, and denote the restriction of $\sigma$ to $E$ by $\sigma_{E}$. We assume in the following that $E \cap \sigma E$ is a function field, and that $E$ and $\sigma E$ are linearly disjoint over $E \cap \sigma E$ for every $\sigma \in U_{1}$. The latter will, for example, be the case if at least one of $E$ and $\sigma E$ is Galois over $E \cap \sigma E$.

Theorem 20. Abbreviating $Z=\operatorname{Con}_{E / E^{V}}\left(\mathrm{~N}_{E / E^{V}}(\operatorname{ker} \phi)\right)$, we have

$$
[C: E](V: W) Z \subseteq \sum_{\substack{\sigma \in U_{1} / / V \\ \sigma \neq 1}}[C: E \sigma E](V: W) \operatorname{Con}_{E / E \cap \sigma E}\left(\mathrm{~N}_{\sigma E / E \cap \sigma E}\left(\sigma_{E}(Z)\right)\right)
$$


Proof. The extensions $C / C^{U_{1}}$ and $E / E^{V}$ are Galois, with groups $U_{1}$ and $V / W$ respectively. We have

$$
\begin{gathered}
\operatorname{Con}_{C / C^{U_{1}}} \circ \mathrm{N}_{C / C^{U_{1}}}=\sum_{\sigma \in U_{1}} \sigma, \\
\sigma \circ \operatorname{Con}_{C / E}=\operatorname{Con}_{C / \sigma E} \circ \sigma_{E}, \\
\sum_{\tau \in V} \tau_{E}=[(V: W)] \circ \operatorname{Con}_{E / E^{V}} \circ \mathrm{N}_{E / E^{V}},
\end{gathered}
$$

for any $\sigma \in U_{1}$. Using Lemma 16 in the fifth equation, we obtain $\mathrm{N}_{C / E} \circ \operatorname{Con}_{C / C^{U_{1}}} \circ \phi=\mathrm{N}_{C / E} \circ \operatorname{Con}_{C / C^{U_{1}}} \circ \mathrm{N}_{C / C^{U_{1}}} \circ \operatorname{Con}_{C / E}$

$$
\begin{aligned}
&= \mathrm{N}_{C / E} \circ\left(\sum_{\sigma \in U_{1}} \sigma\right) \circ \operatorname{Con}_{C / E} \\
&= \sum_{\sigma \in U_{1}} \mathrm{~N}_{C / E} \circ \sigma \circ \operatorname{Con}_{C / E}=\sum_{\sigma \in U_{1}} \mathrm{~N}_{C / E} \circ \operatorname{Con}_{C / \sigma E} \circ \sigma_{E} \\
&= \sum_{\sigma \in U_{1}}[[C: E \sigma E]] \circ \operatorname{Con}_{E / E \cap \sigma E} \circ \mathrm{N}_{\sigma E / E \cap \sigma E} \circ \sigma_{E} \\
&= \sum_{\sigma \in U_{1} / / V}[[C: E \sigma E]] \circ \operatorname{Con}_{E / E \cap \sigma E} \circ \mathrm{N}_{\sigma E / E \cap \sigma E} \circ \sigma_{E} \circ\left(\sum_{\tau \in V} \tau_{E}\right) \\
&= \sum_{\sigma \in U_{1} / / V}[[C: E \sigma E]] \circ \operatorname{Con}_{E / E \cap \sigma E} \circ \mathrm{N}_{\sigma E / E \cap \sigma E} \circ \sigma_{E} \\
& \quad \circ[(V: W)] \circ \operatorname{Con}_{E / E} \circ \mathrm{N}_{E / E^{V}} .
\end{aligned}
$$

If $x \in \operatorname{ker} \phi$ and $z=\operatorname{Con}_{E / E^{V}}\left(\mathrm{~N}_{E / E^{V}}(x)\right)$, writing the summand for $\sigma=1$ separately, we thus have

$$
\begin{aligned}
& \mathrm{N}_{C / E}\left(\operatorname{Con}_{C / C} U_{1}(\phi(x))\right) \\
& =[C: E](V: W) \cdot z+\sum_{\substack{\sigma \in U_{1} / / V \\
\sigma \neq 1}}[C: E \sigma E](V: W) \operatorname{Con}_{E / E \cap \sigma E}\left(\mathrm{~N}_{\sigma E / E \cap \sigma E}\left(\sigma_{E}(z)\right)\right) \\
& =0,
\end{aligned}
$$

thereby proving equation (21) and the theorem.

Proposition 23. Assume that $V$ is normal in $U_{1}$. Then $\sigma\left(E^{V}\right)=(\sigma E)^{V}$, and $E^{V} \cap \sigma E^{V}=$ $(E \cap \sigma E)^{V}$ is a function field for every $\sigma \in U_{1}$. Furthermore, if the kernel of the restriction map $V \longrightarrow \operatorname{Aut}(E)$ is equal to the kernel of the restriction map $V \longrightarrow \operatorname{Aut}(E \cap \sigma E)$, then $E^{V}$ and $\sigma E^{V}$ are linearly disjoint over $E^{V} \cap \sigma E^{V}$.

Proof. Since $V$ is normal in $U_{1}$, it is an automorphism group of $E$ and $\sigma E$, and we have $\sigma\left(E^{V}\right)=(\sigma E)^{V}$. Thus

$$
E^{V} \cap \sigma\left(E^{V}\right)=E^{V} \cap(\sigma E)^{V} \subseteq(E \cap \sigma E)^{V}
$$

because $E^{V} \cap(\sigma E)^{V}$ is contained in $E \cap \sigma E$ and fixed by $V$. Conversely, $(E \cap \sigma E)^{V} \subseteq E^{V}$ and $(E \cap \sigma E)^{V} \subseteq(\sigma E)^{V}$, and hence

$$
(E \cap \sigma E)^{V} \subseteq E^{V} \cap(\sigma E)^{V} ;
$$

in conclusion

$$
E^{V} \cap \sigma E^{V}=(E \cap \sigma E)^{V}
$$


By Galois theory, $E \cap \sigma E$ is of finite degree over $E^{V} \cap \sigma E^{V}$ since $V$ is finite, and thus $E^{V} \cap \sigma E^{V}$ is a function field because $E \cap \sigma E$ is a function field.

The group $W$ is the kernel of the restriction map $V \longrightarrow \operatorname{Aut}(E)$, and is normal in $V$. Furthermore, $\sigma W \sigma^{-1} \subseteq V$ for any $\sigma \in U_{1}$, and $W \sigma W \sigma^{-1} \subseteq \operatorname{ker}(V \longrightarrow \operatorname{Aut}(E \cap \sigma E))=W$, where the last equation holds by assumption. It follows that $\sigma W \sigma^{-1}=W$, and that $W$ is normal in $U_{1}$.

We have $E^{V} \sigma E^{V} \subseteq(E \sigma E)^{V}$, and we want to show equality. Extension $E \sigma E /(E \sigma E)^{V}$ is Galois with group $V / W$, using the fact that $\operatorname{ker}(V \longrightarrow \operatorname{Aut}(E \sigma E))=W \cap \sigma W \sigma^{-1}=W$. Thus

$$
(V: W)=\left[E \sigma E:(E \sigma E)^{V}\right] \leqslant\left[E \sigma E: E^{V} \sigma E^{V}\right] .
$$

On the other hand, we obtain $(V: W) \geqslant\left[E \sigma E: E^{V} \sigma E^{V}\right]$ as follows. We have $E=E^{V}(E \cap \sigma E)$, because $E^{V}(E \cap \sigma E)$ is an intermediate field of the Galois extension $E / E^{V}$ with group $V / W$, and its fix group is $W / W$, using the fact that $W=\operatorname{ker}(V \longrightarrow$ $\operatorname{Aut}(E \cap \sigma E))$. Similarly, $\sigma E=\sigma E^{V}(E \cap \sigma E)$. It follows that $E \sigma E=E^{V} \sigma E^{V}(E \cap \sigma E)$. Then

$$
\begin{aligned}
(V: W) & =\left[E \cap \sigma E:(E \cap \sigma E)^{V}\right] \\
& \geqslant\left[E^{V} \sigma E^{V}(E \cap \sigma E): E^{V} \sigma E^{V}(E \cap \sigma E)^{V}\right] \\
& =\left[E \sigma E: E^{V} \sigma E^{V}\right],
\end{aligned}
$$

as desired. We see that $\left[(E \sigma E)^{V}: E^{V} \sigma E^{V}\right]=1$, and thus $(E \sigma E)^{V}=E^{V} \sigma E^{V}$.

The linear disjointness of $E^{V}$ and $\sigma E^{V}$ over $E^{V} \cap \sigma E^{V}$ now follows, because $E$ and $\sigma E$ are linearly disjoint over $E \cap \sigma E$ and the indices $\left[E: E^{V}\right],\left[\sigma E: \sigma E^{V}\right],\left[E \sigma E: E^{V} \sigma E^{V}\right]$ and $\left[E \cap \sigma E: E^{V} \cap \sigma E^{V}\right]$ are all equal to $(V: W)$, observing that $(E \sigma E)^{V}=E^{V} \sigma E^{V}$ and $(E \cap \sigma E)^{V}=E^{V} \cap \sigma E^{V}$.

THEOREM 24. Under the assumptions of Proposition 23, we have

$$
\left[C^{V}: E^{V}\right] \cdot \operatorname{ker} \phi^{V} \subseteq \sum_{\substack{\sigma \in U_{1} / V \\ \sigma \neq V}} \operatorname{Con}_{E^{V} / E^{V} \cap \sigma E^{V}}\left(\mathcal{C l} l^{0}\left(E^{V} \cap \sigma E^{V}\right)\right),
$$

and the kernel of $\phi$ is contained in the preimage of the right-hand side under the map $[[C: E]] \circ \mathrm{N}_{E / E^{V}}$.

Proof. By Proposition 23, we can apply Theorem 20, replacing $\phi$ by $\phi^{V}$ and $V$ by $\{1\}$. Then $W=\{1\}$ and relation (25) follows from Theorem 20, relation (21). Observing that $[C: E](V: W)=\# V\left[C^{V}: E^{V}\right]$, and hence that $\left[C^{V}: E^{V}\right] \# W=[C: E]$, we obtain

$$
\left[\left[C^{V}: E^{V}\right] \# W\right] \circ \mathrm{N}_{E / E^{V}}=[[C: E]] \circ \mathrm{N}_{E / E^{V}} \text {. }
$$

The statement about the kernel of $\phi$ then follows from (25) and Theorem 18.

\section{L-polynomials}

In this section, we prove a general theorem about the $L$-polynomials of certain subfields of a Galois extension of global function fields with Galois group a semidirect product. The theorem and its corollary are used in the proofs of Theorems 12 and 15. We remark that the $L$-polynomial of a global function field is the characteristic polynomial of Frobenius with the coefficients in reverse order. 
Let $G$ be a finite subgroup of $\operatorname{Aut}(C)$, and let $H$ and $U_{1}$ be subgroups of $G$ such that $H$ is normal in $G, H \cap U_{1}=\{1\}$ and $G=H U_{1}$. The subgroup $U_{1}$ operates on $H$ by conjugation. Assume further that $H$ is elementary abelian of prime exponent $l$, and let $\left\{H_{v}: v \in I\right\}$ be a system of representatives under the operation of $U_{1}$ on the subgroups of $H$ of index $l$ for some index set $I$. Let $U_{1 v}$ be the largest subgroup of $U_{1}$ that leaves $H_{v}$ invariant, and for any subgroup $A$ of $G$ denote the degree of the exact constant field of $C^{A}$ over that of $C^{G}$ by $d_{C^{A}}$.

THEOREM 26. Under the above assumptions, the L-polynomials satisfy

$$
L_{C^{U_{1}}}\left(t^{d} C^{U_{1}}\right) / L_{C^{G}}(t)=\prod_{v \in I} L_{C^{H_{\nu} U_{1 v}}}\left(t^{d} C^{H_{\nu} U_{1 v}}\right) / L_{C^{H U_{1 v}}}\left(t^{d_{C} H U_{1 v}}\right) .
$$

Proof. Since conjugation by elements of $U_{1 v}$ maps $H_{v}$ and $H$ to themselves, $H_{v} U_{1 v}$ and $H U_{1 v}$ are subgroups of $G$. Furthermore, $H U_{1 v}$ is in fact the normaliser of $H_{v}$ in $G$, because $G=H U_{1}$ and $H_{v}$ is normal in $H$. The factor group $H U_{1 v} / H_{v}$ is then a semidirect product of $H / H_{v}$ and $H_{v} U_{1 v} / H_{v}$.

We start with a statement on (non-abelian) characters. The following notation is used: principal characters are denoted by 1 , induced characters are prefixed by 'ind' with the subgroup and group as subscript and superscript, and $\chi^{(v)}$ denotes the character obtained by lifting a character $\chi$ of $H U_{1 v} / H_{v}$ to $H U_{1 v}$. We claim that

$$
\operatorname{ind}_{U_{1}}^{G}(1)-1=\sum_{v \in I} \operatorname{ind}_{H U_{1 v}}^{G}\left(\left(\operatorname{ind}_{H_{v} U_{1 v} / H_{v}}^{H U_{1 v} / H_{v}}(1)-1\right)^{(v)}\right) .
$$

We postpone the proof of (28) and continue to prove (27). Using Artin $L$-series and their functorial properties (see [22, VII.10]), it is straightforward to obtain

$$
\begin{aligned}
& \zeta_{C^{U_{1}}}(s) / \zeta_{C^{G}}(s)=L\left(C / C^{U_{1}}, 1, s\right) / L\left(C / C^{G}, 1, s\right) \\
& =L\left(C / C^{G}, \operatorname{ind}_{U_{1}}^{G}(1)-1, s\right) \\
& =\prod_{\nu \in I} L\left(C / C^{G}, \operatorname{ind}_{H U_{1 v}}^{G}\left(\left(\operatorname{ind}_{H_{\nu} U_{1 v} / H_{v}}^{H U_{u_{v}} / H_{v}}(1)-1\right)^{(v)}\right), s\right) \\
& =\prod_{\nu \in I} L\left(C / C^{H U_{1 v}},\left(\operatorname{ind}_{H_{\nu} U_{1 v} / H_{v}}^{H U_{1_{v}} / H_{\nu}}(1)-1\right)^{(v)}, s\right) \\
& =\prod_{\nu \in I} L\left(C^{H_{v}} / C^{H U_{1 \nu}}, \text { ind }_{H_{\nu} U_{1 v} / H_{\nu}}^{H U_{1 v} / H_{v}}(1)-1, s\right) \\
& =\prod_{\nu \in I} L\left(C^{H_{v}} / C^{H_{\nu} U_{1 v}}, 1, s\right) / L\left(C^{H_{\nu}} / C^{H U_{1 v}}, 1, s\right) \\
& =\prod_{\nu \in I} \zeta_{C^{H_{\nu} U_{1 v}}}(s) / \zeta_{C^{H U_{1 v}}}(s) .
\end{aligned}
$$

Let $q$ denote the cardinality of the exact constant field of $C^{G}$. The switch from the $s$-definition to the $t$-definition of the Zeta function of a global function field over the full constant field of $q^{d}$ elements happens by composing $\zeta^{(t)}=\zeta^{(s)} \circ\left(-\log _{q^{d}}\right)$. Observing that $\log _{q^{d}}\left(t^{d}\right)=\log _{q}(t)=-s$, we obtain (under a slight abuse of notation)

$$
\zeta_{C^{U_{1}}}\left(t^{d_{C} U_{1}}\right) / \zeta_{C} G(t)=\prod_{\nu \in I} \zeta_{C^{H_{\nu} U_{1 \nu}}}\left(t^{d_{C} H_{\nu} U_{1 v}}\right) / \zeta_{C^{H U_{1 \nu}}}\left(t^{d_{C}}{ }^{H U_{1 v}}\right) .
$$


In general, $\zeta^{(t)}\left(t^{d}\right)=L\left(t^{d}\right) /\left(\left(1-q^{d} t^{d}\right)\left(1-t^{d}\right)\right)$, where $L(t)$ is the $L$-polynomial of a global function field over the full constant field of $q^{d}$ elements. Furthermore, $L\left(a^{d}\right) \neq 0$ for $|a|=1$ or $|a|=q^{-1}$. Bringing the denominators $\left(1-q^{d} t^{d}\right)\left(1-t^{d}\right)$ to one side and the $L$-polynomials to the other in (29), and comparing zeros and poles on each side, we see that the denominators of the Zeta functions cancel out, so that we obtain (27).

It remains to prove (28). If $A$ is a finite group, then we denote by $\mathbb{C}[A]$ the regular representation space with character $r_{A}$, by $I_{A}=\left\{\sum_{a \in A} \lambda_{a} a: \sum_{a \in A} \lambda_{a}=0\right\}$ the augmentation representation space with character $r_{A}-1$, and by $\mathbb{C N}_{A}$ for $\mathrm{N}_{A}=\sum_{a \in A} a$ the trivial representation space with character 1 of $A$.

In the discussion that follows, all the modules will be left modules. Assume that $N$ is a normal subgroup of a finite group $A$ with complement $B$ (such that $N B=A$ is a semidirect product). The subgroup $B$ operates by conjugation on $N$, and from this the $N$-module structure of $\mathbb{C}[N]$ can be extended to an $A$-module structure, via $(n b) x=n b x b^{-1}$ for $n \in N, b \in B$ and $x \in \mathbb{C}[N]$. As $A$-modules, we have

$$
\mathbb{C}[N] \cong I_{N} \oplus \mathbb{C} N_{N} \quad \text { and } \quad \mathbb{C}[N] \cong \mathbb{C}[A] \otimes \mathbb{C}[B] \mathbb{C N}_{B}=\operatorname{Ind}_{B}^{A}\left(\mathbb{C} N_{B}\right),
$$

where the second isomorphism is given by $x \mapsto x \otimes \mathrm{N}_{B}$; we observe that $N$ is a set of coset representatives for $B$ in $A$, and that $B$ operates trivially on $\mathrm{N}_{B}$. The equality holds by definition. We apply these observations to our case, and obtain as $G$-modules

$$
I_{H} \oplus \mathbb{C N}_{H} \cong \operatorname{Ind}_{U_{1}}^{G}\left(\mathbb{C} N_{U_{1}}\right),
$$

and as $H U_{1 v} / H_{\nu}$-modules

$$
I_{H / H_{v}} \oplus \mathbb{C N}_{H / H_{v}} \cong \operatorname{Ind}_{H_{v} U_{1 v} / H_{v}}^{H U_{1 v} / H_{v}}\left(\mathbb{C N}_{H_{\nu} U_{1 v} / H_{v}}\right),
$$

taking the remarks at the beginning of the proof into account.

Next, let $\left\{H_{v}: v \in J\right\}$ be the set of all subgroups of $H$ of index $l$ for some index set $J$ such that $I \subseteq J$. Since $H$ is elementary abelian, we have

$$
r_{H}-1=\sum_{\nu \in J}\left(r_{H / H_{\nu}}-1\right)^{(*)},
$$

where $(*)$ denotes the pull-back character with respect to $H \longrightarrow H / H_{\nu}$. This can be seen as follows. The characters $r_{H}$ and $r_{H / H_{\nu}}$ are the sums of all the irreducible characters of $H$ and $H / H_{\nu}$ respectively, and these characters are homomorphisms into $\mu_{l}$, the group of $l$ th roots of unity in $\mathbb{C}$. Now every non-trivial irreducible character of $H$ has precisely one of the $H_{\nu}$ as kernel, due to the assumptions on $H$, and is hence the pull-back of a uniquely defined non-trivial character of $H / H_{\nu}$. Grouping together irreducible characters with the same kernel and summing up yields (33).

Using (33), we see that, as $H$-modules,

$$
I_{H} \cong \sum_{\nu \in J} I_{H / H_{v}}{ }^{(*)}
$$

where $I_{H / H_{v}}{ }^{(*)}$ is $I_{H / H_{v}}$ viewed as an $H$-module. In fact, $I_{H / H_{v}}$ is even an $H U_{1 v}$-module, and we denote this by $I_{H / H_{v}}{ }^{(v)}$. Let $\mathbb{C}[H]^{H_{\nu}}$ and $I_{H}^{H_{v}}$ be, respectively, the submodules of $\mathbb{C}[H]$ and $I_{H}$ fixed by $H_{\nu}$. For a system of coset representatives $H / / H_{\nu}$, it holds that

$$
\mathbb{C}[H]^{H_{\nu}}=\left\{\sum_{h \in H / / H_{\nu}} \lambda_{h} h \mathrm{~N}_{H_{\nu}}: \lambda_{h} \in \mathbb{C}\right\}
$$


and

$$
I_{H}^{H_{v}}=\left\{\sum_{h \in H / / H_{v}, h \notin H_{v}} \lambda_{h} h \mathrm{~N}_{H_{v}}: \lambda_{h} \in \mathbb{C}\right\} ;
$$

hence $\mathbb{C}[H]^{H_{v}} \cong \mathbb{C}\left[H / H_{v}\right]$ as $H U_{1 v^{-}}$and $H U_{1 v} / H_{v}$-modules and $I_{H}^{H_{v}} \cong I_{H / H_{v}}{ }^{(v)}$ as $H U_{1 v}$-modules. The images of the $I_{H / H_{v}}{ }^{(*)}$ under (34) in $I_{H}$ are contained in (and are in fact equal to) the $I_{H}^{H_{\nu}}$, because $H_{v}$ operates trivially on these images and $I_{H / H_{v}}{ }^{*} \cong I_{H}^{H_{v}}$. Equation 34 translates into the inner direct sum

$$
I_{H}=\sum_{v \in J} I_{H}^{H_{v}} .
$$

Now $U_{1}$ operates on the left- and right-hand sides of (35) by conjugation, permuting the direct summands. More precisely, we have

$$
\sigma I_{H}^{H_{\nu}}=I_{H}^{\sigma H_{\nu} \sigma^{-1}} \quad \text { for } \sigma \in U_{1} .
$$

The group $H U_{1 v}$ is the largest subgroup of $G$ that fixes $H_{v}$, and a system $U_{1} / / U_{1 v}$ of coset representatives for $U_{1 v}$ in $U_{1}$, such that $U_{1}=\cup_{\sigma \in U_{1} / / U_{1 \nu}} \sigma U_{1 \nu}$, is also a system of coset representatives for $H U_{1 v}$ in $G$, because $H$ is normal in $G$. From these statements and the definitions of $I$ and $J$, we find that, as $G$-modules,

$$
\operatorname{Ind}_{H U_{1 v}}^{G}\left(I_{H}^{H_{v}}\right) \cong \sum_{\sigma \in U_{1} / / U_{1 v}} I_{H}^{\sigma H_{\nu} \sigma^{-1}}
$$

and

$$
I_{H} \cong \sum_{\nu \in I} \operatorname{Ind}_{H U_{1 \nu}}^{G}\left(I_{H}^{H_{\nu}}\right)
$$

all sums being direct. Substituting $I_{H / H_{v}}{ }^{(v)}$ for $I_{H}^{H_{v}}$ in (36) gives

$$
I_{H} \cong \sum_{\nu \in I} \operatorname{Ind}_{H U_{1 v}}^{G}\left(I_{H / H_{v}}{ }^{(v)}\right)
$$

as $G$-modules. Combining (31), (32) and (37), we obtain (28).

COROLlARY 38. The genera satisfy the equation

$$
d_{C^{U_{1}}} g_{C^{U_{1}}}-g_{C^{G}}=\sum_{\nu \in I}\left(d_{C^{H_{\nu} U_{1}}} g_{C^{H_{\nu} U_{1 \nu}}}-d_{C^{H U_{1}}} g_{C^{H U_{1 \nu}}}\right) .
$$

Proof. This follows if we take the degrees on both sides of (27), since the degree of an $L$-polynomial is twice the genus.

The proof of Theorem 26 simplifies greatly for $U_{1}=\{1\}$ using (28) only in the form (33), and then gives an alternative, short proof of the genus formula given in [12].

\section{Conclusion}

Using statements for extensions with certain automorphism groups, we have investigated the Weil descent methodology for general Artin-Schreier extensions. We have given a formula for the resulting genera and the zeta function, and have discussed the kernel of the norm-conorm homomorphism. Our results apply in particular to hyperelliptic and elliptic 
curves in characteristic two. We have obtained a generalisation of the GHS attack, showing more elliptic curves to be vulnerable. The precise practical implications of the new construction have yet to be determined. We have also given a brief discussion of index calculus in the divisor class groups of the resulting curves, and of further possible generalisations and applications of our techniques.

Acknowledgements. The author thanks C. Diem for helpful comments. This work was supported by the EPSRC.

\section{References}

1. S. Arita, 'Weil descent of elliptic curves over finite fields of characteristic three', Advances in cryptology-ASIACRYPT 2000, Kyoto, Japan, Lecture Notes in Comput. Sci. 1976 (ed. T. Okamoto, Springer, New York, 2000) 248-258. 184

2. E. Artin and J. TATE, Class field theory (Benjamin, New York, 1968). 169

3. I. Blake, G. Seroussi and N. Smart, Elliptic curves in cryptography, London Math. Soc. Lecture Note Ser. 265 (Cambridge University Press, Cambridge, 1999). 178

4. D. Boneh and M. Franklin, 'Identity-based encryption from the Weil pairing', Advances in cryptology - CRYPTO 2001, Lecture Notes in Comput. Sci. 2139 (ed. J. Kilian, Springer, New York, 2001) 213-229. 184

5. M. Ciet, J.-J. Quisquater and F. SiCA, 'A secure family of composite finite fields suitable for fast implementation of elliptic curve cryptography', [23] 108-116. 168

6. C. DiEm, 'The GHS-attack in odd characteristic', J. Ramanujan Math. Soc 18 (2003) 1-32. 168, 169

7. G. Frey, 'How to disguise an elliptic curve', Talk at ECC'98, Waterloo; available at http://www.cacr.math. uwaterloo.ca/conferences/1998/ecc98/frey.ps. 167

8. G. FREY and H.-G. RÜCK, 'A remark concerning $m$-divisibility and the discrete logarithm in the divisor class group of curves', Math. Comp. 62 (1994) 865-874. 184

9. S. Galbraith, 'Weil descent of Jacobians', WCC2001 International workshop on coding and cryptography, Electron. Notes Discrete Math. 6 (ed. D. Augot and C. Carlet, Elsevier, Amsterdam/Paris, 2001). 168

10. S. Galbraith and N. P. Smart, 'A cryptographic application of Weil descent', Cryptography and coding, Lecture Notes in Comput. Sci. 1746 (ed. M. Walker, Springer, New York, 1999) 191-200. 167

11. S. Galbraith, F. Hess and N. P. Smart, 'Extending the GHS Weil descent attack', Advances in cryptology - EUROCRYPT 2002, Lecture Notes in Comput. Sci. 2332 (ed. L. R. Knudsen, Springer, New York, 2002) 29-44. 168, 178, 183

12. A. Garcia and H. Stichtenoth, 'Elementary abelian $p$-extensions of algebraic function fields', Manuscripta Math. 72 (1991) 67-79. 190

13. P. Gaudry, F. Hess and N. P. Smart, 'Constructive and destructive facets of Weil descent on elliptic curves', J. Cryptology 15 (2002) 19-46. 167, 168, 169, 171, 174, $176,177,178$

14. F. Hess, 'Zur Divisorenklassengruppenberechnung in globalen Funktionenkörpern', PhD Thesis, Technische Universität Berlin, 1999. 181 
15. F. Hess, 'Computing Riemann-Roch spaces in algebraic function fields and related topics', J. Symbolic Comput. 33 (2002) 425-445. 180

16. F. Hess, 'Computing relations in divisor class groups of algebraic curves over finite fields', submitted; http://www.math.tu-berlin.de/ hess/dlog.ps.gz. 181

17. IETF (Internet Engineering Task Force), 'The Oakley key determination protocol', IETF RFC 2412; http://www. ietf .org/rfc/rfc2412.txt. 167, 178

18. M. Jacobson, A. Menezes and A. Stein, 'Solving elliptic curve discrete logarithm problems using Weil descent', J. Ramanujan Math. Soc 16 (2001) 231-260. 167, 178

19. M. Maurer, A. Menezes and E. Teske, 'Analysis of the GHS Weil descent attack on the ECDLP over characteristic two finite fields of composite degree', [23] 195-213. 168,176

20. A. Menezes and M. Qu, 'Analysis of the Weil descent attack of Gaudry, Hess and Smart', Progress in cryptology - CT-RSA 2001, Lecture Notes in Comput. Sci. 2020 (ed. D. Naccache, Springer, New York, 2001) 308-318. 167, 178

21. A. Menezes, T. Oкамотo and S. Vanstone, 'Reducing elliptic curve logarithms to logarithms in a finite field', IEEE Trans. Inform. Theory 39 (1993) 1639-1646. 184

22. J. NeuKIRCH, Algebraic number theory (Springer, New York, 1999). 169, 188

23. C. Pandu Rangan and C. Ding (eds), Progress in cryptology-INDOCRYPT 2001, Chennai, India, Lecture Notes in Comput. Sci. 2247 (Springer, New York, 2001). 191, 192

24. N. P. SMART, 'How secure are elliptic curves over composite extension fields?' Advances in cryptology - EUROCRYPT 2001, Lecture Notes in Comput. Sci. 2045 (ed. B. Pfitzmann, Springer, New York, 2001) 30-39. 167

25. H. Stichtenoth, Algebraic function fields and codes (Springer, Berlin, 1993). 169, 174

26. N. ThÉriault, 'Weil descent attack for Artin-Schreier curves', submitted; available at http://www.math.toronto.edu/nicolast/weildescent.pdf. 168

F. Hess hess@math.tu-berlin.de

http://www . math.tu-berlin.de/ hess

Technical University of Berlin

Faculty II - Institute of Mathematics, MA8-1

Straße des 17. Juni 136

10623 Berlin

Germany 